\title{
The FBW7-MCL-1 axis is key in M1 and M2 macrophage-related colon cancer cell progression: validating the immunotherapeutic value of targeting $\mathrm{Pl} 3 \mathrm{~K} \gamma$
}

\author{
Yeo Song Lee $\mathbb{B}^{1,2}$, Su Jeong Song ${ }^{2}$, Hye Kyung Hong ${ }^{2}$, Bo Young $\mathrm{Oh}^{3}$, Woo Yong Lee ${ }^{4,5}$ and Yong Beom Cho ${ }^{4,5}$
}

\begin{abstract}
Colorectal cancer is a devastating disease with a low 5-year survival rate. Recently, many researchers have studied the mechanisms of tumor progression related to the tumor microenvironment. Here, we addressed the prognostic value of tumor-associated macrophages (TAMs) using a total of 232 CRC patient tissue samples and investigated the mechanisms underlying TAM-related colon cancer progression with respect to PI3KY regulation using in vitro, in vivo, and ex vivo approaches. Patients with $\mathrm{M} 2 / \mathrm{M} 1<3$ had significantly improved progressionfree survival and overall survival compared with patients with $M 2 / M 1>3$. M1 and $M 2$ macrophages elicited opposite effects on colon cancer progression via the FBW7-MCL-1 axis. Blocking macrophage PI3KY had cytotoxic effects on colon cancer cells and inhibited epithelial-mesenchymal transition features by regulating the FBW7MCL-1 axis. The results of this study suggest that macrophage PI3Ky may be a promising target for immunotherapy in colon cancer.
\end{abstract}

\section{Introduction}

Colorectal cancer (CRC) is one of the most common malignancies worldwide. To improve the survival rates of patients with CRC, many researchers have studied the underlying mechanisms of carcinogenesis related to both the tumor cell itself and the tumor microenvironment. Like most other solid carcinomas, CRC has a microenvironment that is infiltrated by a variety of immune cells, including tumor-associated macrophages (TAMs), monocytes, dendritic cells, natural killer cells, CD4 $4^{+}$ $\mathrm{T}$ cells, and $\mathrm{CD}^{+} \mathrm{T}$ cells ${ }^{1-3}$. Among these immune cells, TAMs are usually the most abundant immune cells in the tumor microenvironment ${ }^{4-6}$. Recruited macrophages adapt to their environment by developing one of two

\footnotetext{
Correspondence: Yong Beom Cho (gscyb@skku.edu)

'Sungkyunkwan University School of Medicine, Seoul, Republic of Korea

${ }^{2}$ Institute for Future Medicine Samsung Medical Center, Seoul, Republic of Korea

Full list of author information is available at the end of the article
}

major polarization phenotypes: M1 (classical) and M2 (alternative). TAMs usually have M2 characteristics associated with poor survival in patients with breast cancer $^{7}$, lung cancer $^{8}$, bladder cancer ${ }^{9}$, and ovarian cancer $^{10}$. In contrast, increased TAMs are positively correlated with favorable outcomes in lung cancer ${ }^{11}$, prostate cancer $^{12}$, and stomach cancer ${ }^{13}$. Furthermore, several studies on CRC cases have shown that TAMs can lead to a favorable prognosis ${ }^{14-19}$. In addition, high macrophage infiltration into the invasive front of colon cancer is correlated with low liver metastasis ${ }^{20}$. Hence, continued investigation on the role of TAMs in CRC progression might lead to improved therapeutic strategies. Class I phoaphoinositide 3-kinase (PI3K) signaling regulates metabolic pathways during inflammation and carcinogenesis $^{21}$. PI3K $\gamma$ is a class IB isoform of PI3K that is abundantly expressed in myeloid cells, including macrophages. However, PI3K $\gamma$ is not expressed in cancer cells ${ }^{22}$. Previous studies have implicated PI3K $\gamma$ in the regulation

\section{(c) The Author(s) 2020}

(c) (i) Open Access This article is licensed under a Creative Commons Attribution 4.0 International License, which permits use, sharing, adaptation, distribution and reproduction cc) in any medium or format, as long as you give appropriate credit to the original author(s) and the source, provide a link to the Creative Commons license, and indicate if changes were made. The images or other third party material in this article are included in the article's Creative Commons license, unless indicated otherwise in a credit line to the material. If material is not included in the article's Creative Commons license and your intended use is not permitted by statutory regulation or exceeds the permitted use, you will need to obtain permission directly from the copyright holder. To view a copy of this license, visit http://creativecommons.org/licenses/by/4.0/. 
of the innate immune response during cancer progression through TSC, PDK1, and Akt1 ${ }^{23-26}$. Furthermore, inhibition of macrophage $\mathrm{PI} 3 \mathrm{~K} \gamma$ downregulates pancreatic ductal adenocarcinoma tumor progression and increases survival rates by altering macrophage transcriptional profiles $^{27}$. As shown above, the roles of M1 and M2 TAMs in CRC and their prognostic value remain ambiguous. In addition, the mechanisms underlying TAM-related cancer progression with respect to $\mathrm{PI} 3 \mathrm{~K} \gamma$ regulation are currently unclear.

Therefore, the objectives of this study were (1) to investigate the correlation between TAM subtype and colon cancer cell progression; (2) to determine the mechanism involved in their interaction; (3) to explore the mechanisms underlying TAM-related cancer progression with respect to PI3K $\gamma$ regulation; and (4) to determine the prognostic value of TAM subtype in the CRC microenvironment.

\section{Materials and methods \\ Patients and data collection}

From 2006 to 2007, paraffin-embedded samples of CRC patients were obtained from the Department of Surgery, Samsung Medical Center (Sungkyunkwan University School of Medicine), Seoul, Korea. All patients underwent a surgical procedure for colon or rectal cancer, and their data were prospectively entered into a database. Clinical data were retrospectively collected and analyzed based on medical records and operative notes. This study was approved by the Institutional Review Board (IRB) of Samsung Medical Center (IRB No. 2010-09-017). A total of 232 CRC patients (stage I, $n=32$; stage II, $n=70$; stage III, $n=76$; and stage IV, $n=54$ ) were analyzed for clinical outcomes, including progression-free survival (PFS) and overall survival (OS).

\section{Multiplex tissue microarray (TMA) analysis}

Tissue cores ( $2 \mathrm{~mm}$ in diameter) were carefully transferred to recipient paraffin blocks. Filled recipient blocks were embedded in paraffin and sectioned $(4 \mu \mathrm{m}$ in thickness). TMA slides were serially stained using the Opal Kit (PerkinElmer, Hopkinton, MA, USA) for immunohistochemical (IHC) analysis. In brief, sections were dewaxed by heating at $55^{\circ} \mathrm{C}$ for $30 \mathrm{~min}$ followed by three washes with xylene ( 5 min per wash). The slides were then rehydrated in serial ethanol solutions $(100,90$, and $80 \%$ ) and distilled water (5 min each). Antigen retrieval was achieved using a microwave oven at $100 \%$ power followed by $10 \%$ power in citrate antigen retrieval buffer ( $\mathrm{pH}$ 6.0) for $15 \mathrm{~min}$. Endogenous peroxidase activity was blocked by incubating the slides in $0.3 \%$ hydrogen peroxide at room temperature for $10 \mathrm{~min}$. Background reactivity was removed using universal blocking serum (Dako Diagnostics, Glostrup, Denmark) for $30 \mathrm{~min}$ at room temperature. Slides were then incubated with antibodies specific to CD86 (\#ab53004, Abcam, Cambridge, MA) and CD163 (\#ab17051, Abcam, Cambridge, MA) for $1 \mathrm{~h}$ at room temperature. After primary antibody incubation, the sections were washed with $0.02 \%$ Tris-buffered saline containing $0.1 \%$ Tween 20 (TBST) and incubated with secondary horseradish peroxidase-conjugated antibodies at room temperature for $10 \mathrm{~min}$. The sections were washed three times with TBST, followed by tyramide signal amplification (TSA) using TSA-Cy3 (PerkinElmer, Boston, MA) and TSAFITC (PerkinElmer, Boston, MA) at room temperature for $10 \mathrm{~min}$ according to the manufacturer's instructions. All sections were washed five times with TBST $(5 \mathrm{~min}$ each), followed by DAPI staining (PerkinElmer, Boston, MA). The sections were mounted with Vectashield HardSet medium (Vector, Burlingame, USA). Dried slides were scanned using the PerkinElmer Vectra 3.0 platform at $\times 20$ magnification. Images were subjected to phenotyping and analyzed using inForm Advanced Image Analysis Software (PerkinElmer, Boston, MA). Cells that were positive for CD86 and CD163 were classified as M1 and M2 macrophages, respectively. Cell quantification was performed using Spotfire (TIBCO, Boston, MA).

\section{Cell cultures, macrophage polarization, and reagents}

Human CRC cell lines (HCT116, HT29, LoVo, and SW48), a human monocyte cell line (THP-1), and a mouse colon cancer cell line (CT26) were obtained from American Type Culture Collection (ATCC, Manassas, VA, USA). Cells were cultured in Rosewell Park Memorial Institute (RPMI) 1640 (Gibco, Grand Island, NY, USA) supplemented with $10 \%$ fetal bovine serum (FBS, Gibco) and $1 \%$ penicillin-streptomycin (Gibco, NY, USA) in a $5 \% \mathrm{CO}_{2}$ incubator at $37^{\circ} \mathrm{C}$. According to previous studies $^{28,29}$, to polarize M0 macrophages, THP1 cells $\left(5 \times 10^{6}\right)$ were plated in 100 -phi dishes and stimulated with $320 \mathrm{~nm}$ phorbol 12-myristate 13-acetate (PMA; Sigma, St. Louis, MO, USA), followed by incubation at $37^{\circ} \mathrm{C}$ for $24 \mathrm{~h}$. The culture supernatant was collected and labeled M0 macrophage-conditioned medium (M0 CM). To polarize M1 or M2 macrophages, THP-1 cells were treated with PMA for $6 \mathrm{~h}$, and M1-polarizing reagents $(100 \mathrm{ng} / \mathrm{ml}$ lipopolysaccharide (LPS) plus $20 \mathrm{ng} / \mathrm{ml}$ interferon gamma (IFN- $\gamma$ ); R\&D, Waltham, MA) or M2-polarizing reagents $(20 \mathrm{ng} / \mathrm{ml} \mathrm{IL-}$ 4 plus $20 \mathrm{ng} / \mathrm{ml} \mathrm{IL-13)}$ were added, followed by incubation at $37^{\circ} \mathrm{C}$ for $18 \mathrm{~h}$. The culture supernatant was collected and labeled M1 macrophage CM (M1 CM) or M2 macrophage CM (M2 CM). MG132 (proteasome inhibitor) and PI3K $\gamma$ inhibitors (TG100-115 and IPI549) were purchased from Sigma-Aldrich (St. Louis, 
MO, USA) and Selleckchem (Houston, TX, USA), respectively.

\section{Cell proliferation assay}

The cell proliferation assay was performed in triplicate. A colorimetric assay was performed to determine cell viability by evaluating the metabolic conversion of watersoluble tetrazolium salt (WST)-1 (2-(4-iodophenyl)-3(4-nitrophenyl)-5-(2,4-disulfophenyl)-2H-tetrazolium)) purchased from Roche (Indianapolis, IN, USA). In brief, WST-1 was added directly into culture wells and incubated at $37^{\circ} \mathrm{C}$ for $60-120 \mathrm{~min}$. Absorbance was measured at a wavelength of $450 \mathrm{~nm}$. Three different experiments were conducted for each experimental condition.

\section{Cell lysis and western blot analysis}

To obtain whole-cell extracts, cells were lysed with Proprep buffer (Intron Biotechnology, Seoul, Korea) containing protease inhibitors. Protein extracts $(10-60 \mu \mathrm{g})$ were resolved by sodium dodecyl sulfate polyacrylamide gel electrophoresis and transferred to polyvinylidene difluoride membranes. These membranes were probed with SURVIVIN (\#AF886, R\&D Systems, Waltham, MA), BMI-1 (\#sc-10745, Santa Cruz, CA, USA), Caspase-3 (\#9662, Cell Signaling, MA, USA), PARP (\#9542, Cell Signaling, MA, USA), E-cadherin (\#610181, BD Bioscience, San Jose, CA), N-cadherin (\#4061, Cell Signaling, MA, USA), VIMENTIN (\#sc-32322, Santa Cruz, CA, USA), MMP2 (\#13132, Cell Signaling, MA, USA), phospho AKT (\#4060, Cell Signaling, MA, USA), AKT (\#4691, Cell Signaling, MA, USA), phospho ERK (\#612358, BD Bioscience, San Jose, CA), ERK (\#9102, Cell Signaling, MA, USA), MCL-1 (\#4572, Cell Signaling, MA, USA), FBW7 (\#ab10752, Abcam, Cambridge, MA), and $\beta$-actin (\#3700, Cell Signaling, MA, USA) primary antibodies, followed by incubation with secondary antibodies conjugated to horseradish peroxidase (Santa Cruz Biotechnology, CA, USA). $\beta$-actin was used as a loading control for western blot analysis.

\section{Animals}

Six- to seven-week-old female BALB/c nu/nu and $\mathrm{BALB} / \mathrm{c}$ wild-type mice weighing $15-17 \mathrm{~g}$ at the time of transplantation were used in this study. These mice were obtained from Orient Bio (Seoul, Korea) and maintained under specific pathogen-free conditions. This study was reviewed and approved by the Institutional Animal Care and Use Committee (IACUC) of Samsung Biomedical Research Institute (SBRI). SBRI is a facility that is accredited by the Association for Assessment and Accreditation of Laboratory Animal Care International (AAALAC International). This study abided by the Institute of Laboratory Animal Resources (ILAR) guidelines.

\section{Coculture of polarized macrophages and mouse xenografts}

Polarized M0, M1, and M2 macrophages $\left(4 \times 10^{5}\right.$ cells) were seeded into the lower compartment of a six-well Boyden chamber. HCT116 cells $\left(2.5 \times 10^{5}\right.$ cells $)$ were added into the upper inserts. The cells were cocultured at $37^{\circ} \mathrm{C}$ for 7 days, followed by 3 days of CM treatment. Tumorigenesis was measured using a mouse xenograft model to evaluate the effects of polarized macrophages on cancer growth. In brief, cocultured HCT116 cells were suspended in PBS supplemented with 50\% Matrigel and injected subcutaneously into the flanks of six- to sevenweek-old female BALB/c nu/nu mice. Tumor size was measured twice a week with a caliper. Tumor volume was calculated using the following formula: tumor volume $=$ (short length $\times$ long length $\times$ width)/2. The mice were sacrificed three weeks after transplantation/injection.

\section{RT-PCR and real-time PCR}

Total RNA was extracted using TRIzol reagent (Invitrogen, Carlsbad, CA, USA). Total RNA (500 ng) was then subjected to reverse transcription using a Superscript II reverse transcriptase kit (Invitrogen, Carlsbad, CA, USA) according to the manufacturer's instructions. Primer sequences used for RT-PCR and real-time PCR are listed in Supplementary Table 1. The mRNA level of each gene was normalized to that of GAPDH or $\beta$-actin.

\section{IHC staining}

IHC analysis for Ki67 (\#sc-15402, Santa Cruz, CA, USA) was performed on $4-\mu \mathrm{m}$ frozen sections of obtained mouse xenograft tissues. The tissue sections were deparaffinized with xylene, hydrated in serial dilutions of alcohol, and immersed in $3 \% \mathrm{H}_{2} \mathrm{O}_{2}$. Following antigen retrieval in citrate buffer $(\mathrm{pH}$ 6.0), the tissue sections were incubated with protein-blocking agent (Immunotech, Marseille, France) at room temperature for $10 \mathrm{~min}$ to block nonspecific antibody binding. The sections were then incubated with primary antibody against Ki67 (1:200) in a humidified chamber overnight at $4{ }^{\circ} \mathrm{C}$. After being washed with PBS three times, these sections were incubated with a biotinylated secondary antibody and streptavidin conjugated to horseradish peroxidase (Immunotech, Marseille, France) for $60 \mathrm{~min}$ at room temperature, followed by a PBS wash. Chromogen was developed with liquid 3,30-diaminobenzidine (Immunotech, Marseille, France), followed by counterstaining with Meyer's hematoxylin. The slides were examined under a light microscope.

\section{Cell invasion and wound-healing assay}

Cell invasion assays were performed using uncoated transwell migration chambers (BD Bioscience, San Jose, CA, USA) in 24-well cell culture plates. Cells $\left(5 \times 10^{4} /\right.$ well $)$ 
were loaded into the invasion chamber in serum-free RPMI. The lower chamber contained RPMI supplemented with $10 \%$ FBS as a chemoattractant. The plates were incubated at $37^{\circ} \mathrm{C}$ for $24 \mathrm{~h}$, followed by staining with hematoxylin-eosin and mounting. Cell invasion was imaged using an upright microscope $(\times 200)$. The woundhealing assay was performed using l-Dish 35-mm-high culture inserts (Ibidi, Martinsried, Germany) according to the manufacturer's protocols. In brief, on the day before experimentation, the cells were seeded into each well of the culture inserts and incubated at $37^{\circ} \mathrm{C}$ in a humidified atmosphere with $5 \% \mathrm{CO}_{2}$. After cell attachment, the culture inserts were gently removed using tweezers, and the cells were incubated in macrophage $\mathrm{CM}$ ) for more than $12 \mathrm{~h}$.

\section{Immunofluorescence microscopy}

After incubation with macrophage CM, HT29 cells were washed twice with cold PBS, fixed in methanol at $4{ }^{\circ} \mathrm{C}$ for 10 min, washed with PBS, permeabilized with $0.1 \%$ Triton $\mathrm{X}-100 / \mathrm{PBS}$ for $30 \mathrm{~min}$, and incubated with a primary antibody against E-cadherin in $1 \% \mathrm{BSA} / 0.1 \%$ Triton X$100 / \mathrm{PBS}$ at $4{ }^{\circ} \mathrm{C}$ overnight. After being washed with PBS, immunolabeled proteins were visualized by treatment with fluorescent-conjugated secondary antibodies for $60 \mathrm{~min}$ at room temperature. The cells were washed with PBS, mounted with DAPI mounting medium, sealed with cover slips, and examined using a confocal laser scanning microscope (LSM 780; Carl Zeiss, NY, USA).

\section{Patient-derived cell (PDC) isolation and dissociation}

Surgically resected colon cancer tissues were obtained from patients from Samsung Medical Center (SMC), Seoul, Korea. This study was approved by the Institutional Review Board (IRB) of SMC (IRB No. 20**-0*-017). Tissues were washed three times with $70 \%$ ethanol, followed by cold PBS washes until the supernatant was clear. Next, the tissues were chopped into $\sim 5$ - $\mathrm{mm}$ pieces and further washed with cold PBS. These pieces were then incubated with digestion buffer (Dulbecco's modified Eagle medium with $2.5 \% \mathrm{FBS}, 1 \%$ penicillin/streptomycin (Invitrogen, Carlsbad), $75 \mathrm{U} / \mathrm{mL}$ collagenase type IV (Gibco, Grand Island, NY), and $125 \mu \mathrm{g} / \mathrm{mL}$ dispase type II (Gibco, Grand Island, NY)) at $37^{\circ} \mathrm{C}$ for $30-60 \mathrm{~min}$. Digested cells were then centrifuged at $200 \mathrm{~g}$ for $3 \mathrm{~min}$ to separate adenoma from single cells. Dissociated cells were passed through a $40-\mu \mathrm{m}$ cell strainer and washed several times with PBS. Isolated colon cancer cells were counted and embedded in Matrigel on ice and seeded into 24-well cell culture plates.

\section{Flow cytometry and morphology analysis}

Cells isolated from mouse tumors were incubated with anti-CD16/32 antibody (Fc blocker, \#553142, BD, San Jose, $\mathrm{CA}$ ) to block nonspecific binding and were stained with the following fluorochrome-tagged antibodies: antiCD11b APC-Cy7 (\#557657, BD, San Jose, CA), antiCD11b APC-Cy7 isotype control (\# 400623, Biolegend), anti-F4/80 Alexa 647 (\#565853, BD, San Jose, CA), antiF4/80 Alexa 647 isotype control (\#557690, BD, San Jose, CA), anti-MHC Class II eFluor 450 (\#48-5321-82, Invitrogen, Carlsbad, CA, USA), anti-MHC Class II eFluor 450 isotype control (\#48-4031-80, Invitrogen, Carlsbad, CA, USA), anti-CD206 PE (\#12-2061-82, Invitrogen, Carlsbad, CA, USA), and anti-CD206 PE isotype control (\#12-4031-81, Invitrogen, Carlsbad, CA, USA) antibodies. Stained cells were then analyzed with a Verse flow cytometer and BD FACS Diva software (BD Biosciences, San Jose, CA). Dead cells and doublets were excluded based on forward scatter and side scatter.

\section{Statistical analysis}

Data obtained from xenograft models were analyzed using GraphPad Prism 5.0 software (La Jolla, CA, USA) with one-way analysis of variance (ANOVA) and post hoc analysis (Bonferroni post hoc test). For clinical data analysis, statistical processing was conducted using SPSS version 19.0 software (SPSS Inc., Chicago, IL, USA). Survival rates were estimated using the Kaplan-Meier method and compared by the log-rank test. The data are presented as the mean \pm SD. Statistical significance was considered at $p<0.05$.

\section{Results}

\section{Differentiation of macrophages into particular subtypes}

THP-1 cells were differentiated according to classical conditions (PMA to polarize macrophages and LPS plus IFN- $\gamma$ or IL-4 plus IL-13 to differentiate M1 or M2 macrophages, respectively). THP-1 cells showed a round and partly clustered morphology and grew in suspension. During the polarization process, THP-1 cells underwent morphological changes, including circle-like shape attachment, size increases in M0 and M2 macrophages, and fibroblast-like changes in M1 macrophages (Supplementary Fig. 1A). RT-PCR analysis showed that CCR7 and CXCL9 (two major M1 macrophage markers) were exclusively expressed in M1-differentiated macrophages, whereas CCL17 and CD23 (well-known M2 macrophage markers) were significantly expressed in M2differentiated macrophages (Supplementary Fig. 1B). To further delineate macrophage subtypes, the expression levels of cytokines that are generally responsible for proinflammatory and antiinflammatory responses in polarized macrophage subtypes were evaluated by RTPCR. Proinflammatory cytokines such as $I L-1 \alpha, I L-1 \beta$, $C X C L 10, I L-8$, and $I L-12 \beta$ were upregulated in M1 macrophages compared with M2 macrophages (Supplementary Fig. $1 \mathrm{C}$, upper panel). However, antiinflammatory cytokines such as TGF- $\beta$ and $I L-10$ were upregulated in 


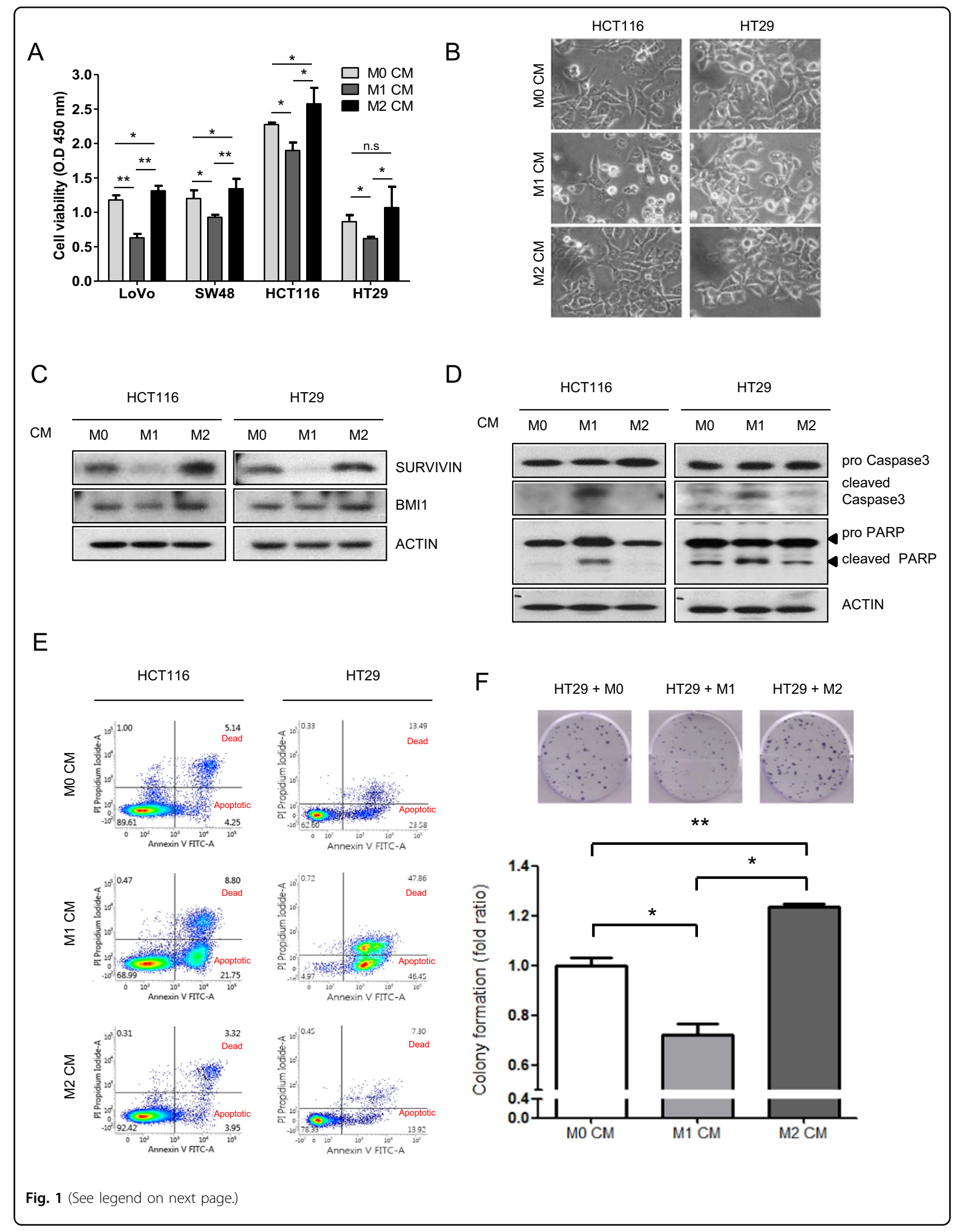


(see figure on previous page)

Fig. 1 Opposite effects of M1 and M2 macrophages on the viability of colon cancer cells. a Viability of four colon cancer cell lines after coculture with macrophage CM for $24 \mathrm{~h}$ based on the WST-1 assay. Error bars are derived from three independent experiments. $\mathbf{b}$ Phase contrast microscopy observation ( $\times 400$ ) of HCT116 and HT29 cells treated with macrophage CM for $24 \mathrm{~h}$. c Expression levels of SURVIVIN and BMI-1 in macrophage CM-treated HCT116 and HT29 cells based on western blotting. $\mathbf{d}$ Extent of caspase-3 activation and cleavage of PARP in cell lysates, as measured by western blotting. e Apoptotic cells were determined by FACS using annexin V/PI double staining after $24 \mathrm{~h}$ of incubation with each macrophage CM with HCT116 and HT29 cells. f The colony formation effect of each macrophage CM on HT29 cells. Representative images of the colony-forming assay (upper panel) and analysis of colony formation rates (lower panel) are shown. The results are presented as the mean value \pm SE. ${ }^{*} P<0.05 ;{ }^{* *} P<0.01 ;{ }^{* *} P<0.001$.

M2 macrophages compared with M1 macrophages (Supplementary Fig. 1C, lower panel).

\section{M1 macrophage CM impedes colon cancer cell viability through apoptosis}

Conditioned media $(\mathrm{CM})$ from differentiated macrophages was collected after $24 \mathrm{~h}$ of polarization, and the effect of CM on viability of normal colon cell (CCD18Co, Supplementary Fig. 1D) and colon cancer cell were investigated. Coculture with M1 CM clearly decreased the viability of LoVo, SW48, HCT116, and HT29 cells. However, M2 CM slightly increased the viability of these colon cancer cells compared with that of M0 CM (Fig. 1a). Among these colon cancer cells, the HCT116 and HT29 cell lines were chosen for further study. As hypothesized, our results revealed that M1 CM had different effects on morphological changes in both HCT116 and HT29 cells (Fig. 1b). The morphology of M1 CM-treated cells changed from a spindle-like to a pebble-like shape. In addition, the cell-to-cell contact of M1 CM-treated cells became less dense than that of M0 CM-treated cells. Furthermore, vacuole formation was significantly increased in M1 CM-treated cells compared with that of M0 CM-treated cells. M1 CM-treated cells also showed morphological characteristics of apoptosis. On the other hand, M0 or M2 CM-treated HCT116 and HT29 cells did not show apoptotic features. Furthermore, the proliferation of M2 CM-treated cells was increased compared with that of MO CM-treated cells. Therefore, apoptosis-related cell death was examined based on apoptosis markers using western blotting and RT-PCR analysis. Western blotting results showed that the expression levels of the antiapoptotic markers SURVIVIN and BMI-1 were attenuated in M1 CMtreated cells. However, M2 CM increased the expression levels of these markers in HCT116 and HT29 cells (Fig. 1c). The results of RT-PCR analysis showed the same expression pattern for SURVIVIN in CM-treated HCT116 and HT29 cells. The expression of $p 21$, a well-known apoptosis marker, was increased in M1 CMtreated cells. However, M2 CM treatment had the opposite effect (Supplementary Fig. 2A). SURVIVIN is an inhibitor of the caspase-related apoptosis pathway.
Therefore, the caspase-related pathway was explored by evaluating caspase-3 and PARP activity. As expected, western blot analysis showed that M1 CM increased caspase- 3 activation, which triggered PARP cleavage. Such PARP activation was shown in M1 CM-treated cell lysates. However, the findings with M2 CM were opposite to those of M0 CM (Fig. 1d). Flow cytometry results confirmed that M1 CM-induced apoptosis, whereas M2 CM inhibited apoptosis in both HCT116 and HT29 cells (Fig. 1e). The results of the colony formation assay showed that M2 CM significantly increased the proliferation of HT29 cells compared with that of M0 CM, whereas M1 CM reduced proliferation (Fig. 1f). These results suggest that $\mathrm{M} 1 \mathrm{CM}$ increases apoptosis, whereas M2 CM inhibits apoptosis compared with M0 CM in HCT116 and HT29 cells.

\section{M2 macrophages promote tumor growth of colon cancer cells in vivo}

Our current data revealed that M1 CM and M2 CM had opposite roles in the proliferation of colon cancer cells in vitro. To confirm these in vitro findings, we evaluated whether M1 and M2 macrophages had opposite effects on colon cancer cells in vivo using a mouse xenograft model. Before transplantation, HCT116 cells were cocultured with macrophages (M0, M1, or M2) for 7 days, followed by incubation with macrophage CM for three days. Consistent with our in vitro results, the in vivo data also showed that the M1 macrophage coculture condition significantly inhibited tumor growth compared with that of M0 or M2 macrophage coculture conditions in a mouse xenograft model that was transplanted with HCT116 cells (Fig. 2a, b). M2 macrophage coculture tended to promote tumor growth more than M0 macrophage coculture, although the difference between the two was not statistically significant. To determine whether M1 and M2 macrophages regulate tumor growth by apoptosis, mRNA, and protein expression levels in mouse subcutaneous tissues were determined. M1 macrophage stimuli downregulated the mRNA expression level of the survival-related marker SURVIVIN but upregulated the mRNA expression level of the apoptosis-related marker $p 21$. On the other hand, M2 macrophage stimuli 
A

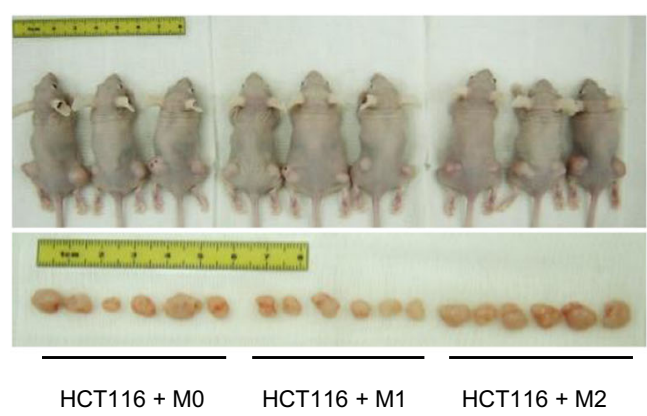

C

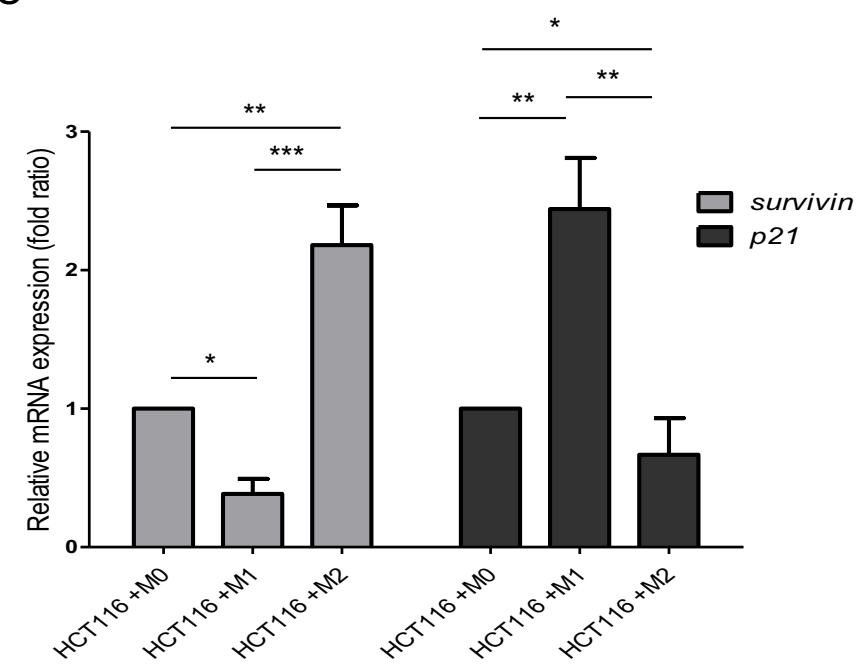

$E$ $\mathrm{HCT} 116+\mathrm{MO} \quad \mathrm{HCT} 116+\mathrm{M} 1$
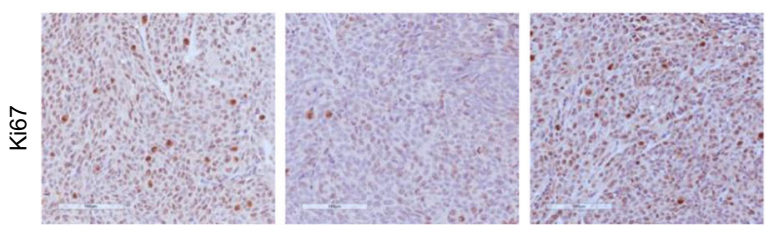

B

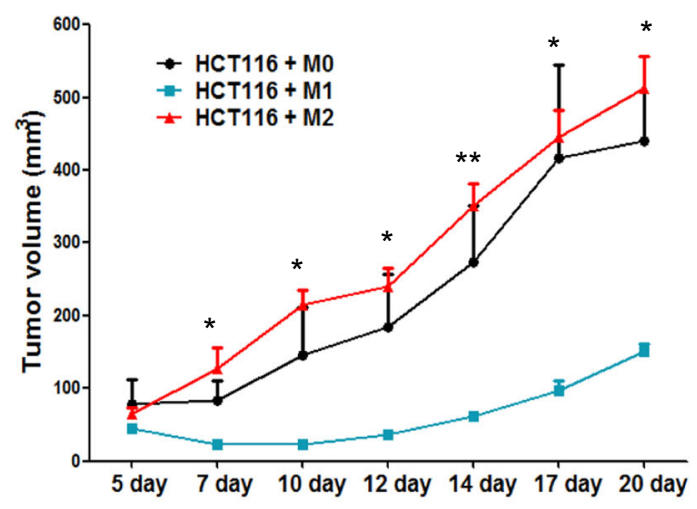

D

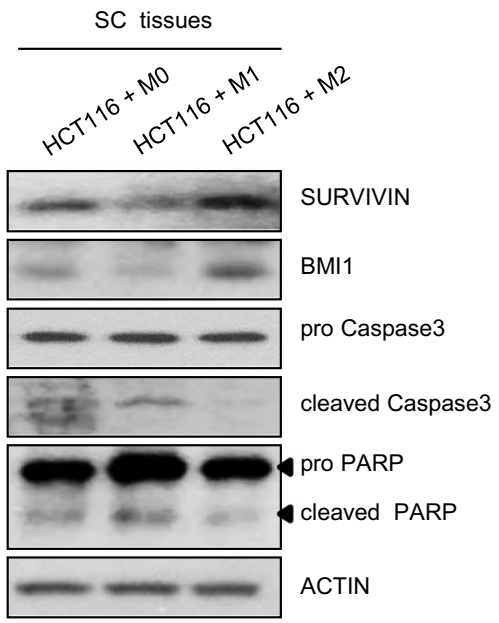

Fig. 2 Opposite effects of $\mathbf{M} 1$ and $M 2$ macrophages on tumor growth. a Tumor images and $\mathbf{b}$ tumor growth 3 weeks after transplantation of HCT116 cells after long-term coculture with differentiated M0, M1, or M2 macrophages (see Materials and Methods). Tumor size was measured 2-3 times a week with a caliper. Tumor volume was calculated using the following formula: tumor volume $=($ short length $\times$ long length $\times$ width $) / 2$. The expression of SURVIVIN or p21 c) and proliferation markers $\mathbf{d}$ in mouse subcutaneous tissues was evaluated by real-time PCR and western blotting, respectively. e Ki67 staining (1:200) of mouse subcutaneous tissues three weeks after subcutaneous injection. Scale bar: $100 \mu \mathrm{m}$. The results shown are presented as the mean \pm SD from three mice per group. Actin was used as a loading control. ${ }^{*} P<0.05 ;{ }^{* *} P<0.01 ;{ }^{* *} P<0.001$.

upregulated SURVIVIN expression but decreased $p 21$ expression (Fig. 2c). Consistent with the mRNA data, the protein expression results also showed that M1 macrophages inhibited tumor growth via caspase-mediated apoptosis (Fig. 2d). Next, IHC staining was performed using Ki67, a well-known proliferation marker used in many cancer tissues. IHC results of Ki67 expression showed that the proliferation of M0/M2 macrophagecocultured HCT116 cells was higher than that of M1 macrophage-cocultured HCT116 cells. In addition, the proliferation of M2 macrophage-cocultured HCT116 cells was higher than that of M0 macrophages (Fig. 2e). 


\section{M2 macrophage CM enhances colon cancer cell migration through epithelial-mesenchymal transition (EMT) regulation}

To assess whether M2 CM induces the EMT in colon cancer cells, invasion and wound-healing assays were conducted using HCT116 and HT29 cells after $24 \mathrm{~h}$ of treatment with each macrophage $\mathrm{CM}$. Both the invasion and migration abilities of M2 CM-treated HCT116 and HT29 cells were increased compared with those of cells treated with M0 CM. On the other hand, M1 CM treatment markedly blocked invasion (Fig. 3a) and migration (Fig. 3c, d) of HCT116 and HT29 cells. Loss of membranous E-cadherin expression enables cancer cell invasion $^{30}$. Therefore, the surface expression of E-cadherin in HT29 cells was analyzed using immunofluorescence staining. As anticipated, E-cadherin expression was decreased in M2 CM-treated cells. However, M1 CM obviously induced E-cadherin expression on the membrane of HT29 cells (Fig. 3b). Protein expression of EMT markers in HCT116 and HT29 cells were also determined by western blotting. E-cadherin expression was increased in M1 CM-treated cells. However, M2 CM impeded Ecadherin expression in comparison with that of M0 CM. Other EMT markers, such as N-cadherin, VIMENTIN, and MMP2, also showed similar features (i.e., M1 CM reduced EMT, while M2 CM-induced EMT in parallel lysates, Fig. 3e). Similar results were observed when the expression level of EMT markers was measured in a mouse xenograft model transplanted with HCT116 cells that were cocultured with different macrophage subtypes (Fig. 3f). These results demonstrate that M2 macrophages induce the EMT phenotype in colon cancer cells.

\section{The FBW7-MCL-1 axis is involved in M1/M2} macrophage-related features of colon cancer cells

The pathways responsible for TAM-induced colon cancer cell proliferation and EMT behavior in HCT116 and HT29 cells, as well as previously obtained mouse subcutaneous tissues, were then determined. MCL- 1 is related to both proliferation and the $\mathrm{EMT}^{31,32}$. Therefore, MCL-1, as a central regulator of these features, was examined in colon cancer cells that were cocultured with macrophages. It has been reported that AKT and ERK enhance the stability of MCL- $1^{33,34}$. Thus, AKT and ERK activities were evaluated by measuring their phosphorylation in macrophage CM-treated colon cancer cells (Fig. 4a) and previously obtained mouse subcutaneous tumors that arose from implanted HCT116 cells that had been cocultured with macrophages (Fig. 4b). The results showed that in HCT116 and HT29 cells and mouse subcutaneous tumors, coculture with M2 macrophages increased AKT and ERK activation, whereas coculture with M1 macrophages reduced their activation compared with coculture with M0 macrophages. The effects of each macrophage phenotype on MCL-1 protein expression were then examined in parallel cell lysates and tumor lysates. Our results revealed that M1 macrophages markedly decreased MCL-1 protein levels. However, coculture with M2 macrophages enhanced MCL-1 protein expression. Previous studies suggested that F-box/ WD repeat-containing protein 7 (FBW7) regulates MCL1 expression via ubiquitination and degradation ${ }^{35}$. Therefore, FBW7 expression was examined in the same cell and tumor lysates. Western blotting results also indicated that FBW7 was involved in MCL-1 stabilization in colon cancer cells (Fig. 4a) and mouse subcutaneous tumors (Fig. 4b) that were cocultured with macrophages. MCL-1 is also regulated at the transcriptional level. Therefore, RT-PCR was conducted to evaluate the gene expression of $M C L-1$ under three CM stimulation conditions. RT-PCR results showed that macrophage subtypes had no effect on MCL-1 mRNA expression. FBW7 mRNA expression was similar to its protein expression pattern. FBW7 expression was relatively high in M1 CMstimulated cells but low in M2 CM-stimulated cells in comparison with that in M0 CM-treated cells (Fig. 4c). To further confirm that MCL-1 was degraded by the ubiquitin-proteasome pathway, MCL-1 expression in macrophage CM-treated HT29 cells that were pretreated with or without a proteasome inhibitor (MG132) was then examined using western blotting. As shown in Fig. 4d, MG132 restored the protein level of MCL-1 in M1 CMtreated HT29 cells. We next examined whether MCL-1 overexpression (Supplementary Fig. 2B) could reverse the effect of macrophage CM in HCT116 cells. MCL-1 overexpression reduced E-cadherin expression after MCL-1-overexpressing HCT116 cells were incubated with M1 CM. Cleaved PARP expression was decreased in each macrophage CM-treated MCL-1-overexpressing HCT116 cell line compared with that of control empty vector-transfected HCT116 cells (Fig. 4e). Furthermore, by using S64846, an MCL-1-specific inhibitor, we found that MCL-1 inhibition in M2 CM-treated cancer cells increased apoptosis and decreased EMT marker expression (Supplementary Fig. 3A). Next, we conducted a cytokine array to discover which cytokines were affected the most by each macrophage CM. Our results showed that M1 CM contained higher levels of the proinflammatory cytokines CXCL11 and IL-1 $\beta$ than M2 CM, whereas M2 CM had higher levels of the antiinflammatory cytokines CCL5 and IL-8 than M1 CM (Supplementary Fig. 3B). IFN- $\gamma$, IL-13, and IL-4 were excluded from analysis because they were used for macrophage polarization.

Our results verified that posttranslational modulation of the FBW7-Mcl-1 axis was an underlying mechanism involved in macrophage-mediated colon cancer cell proliferation and EMT behavior via the activation of AKT and ERK. 

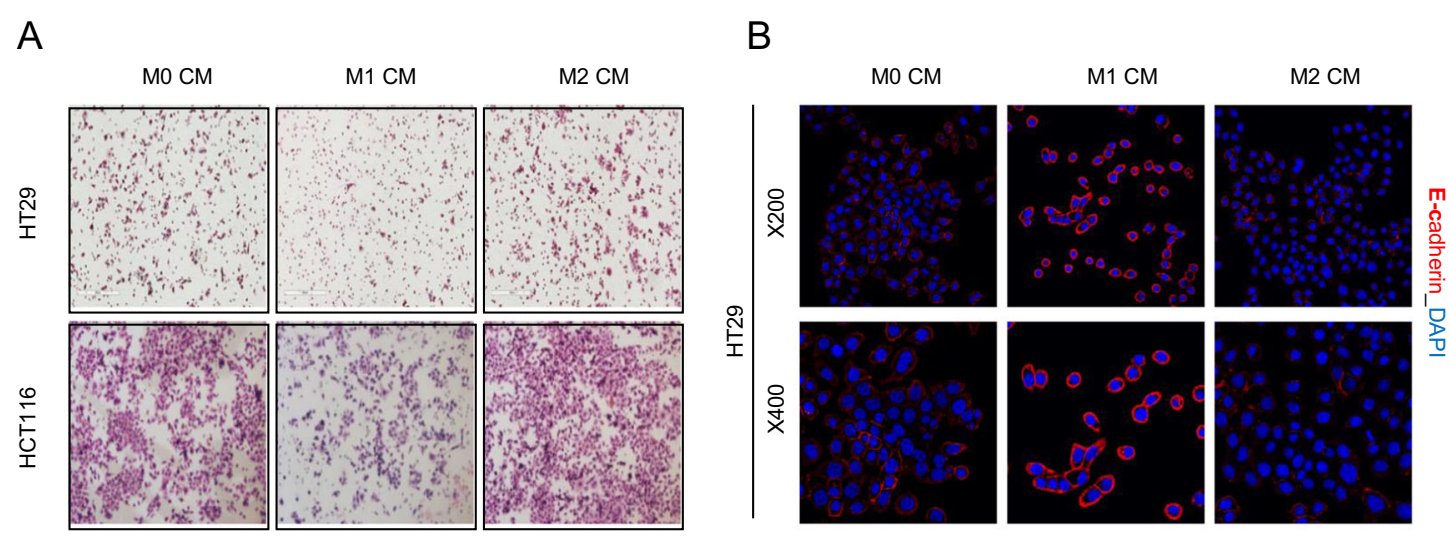

C

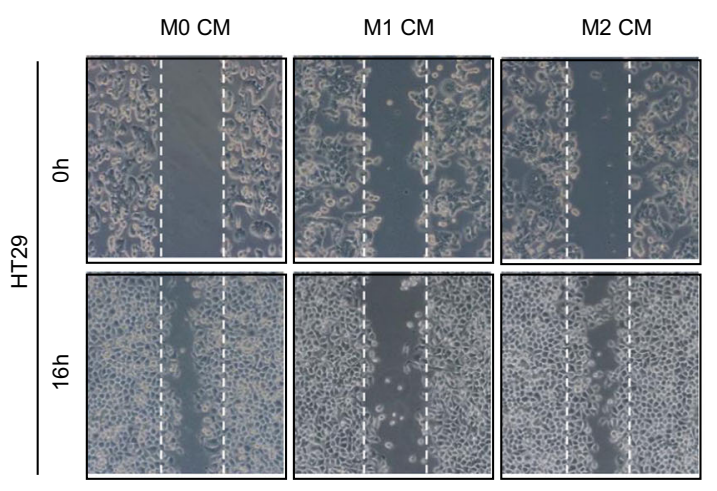

D

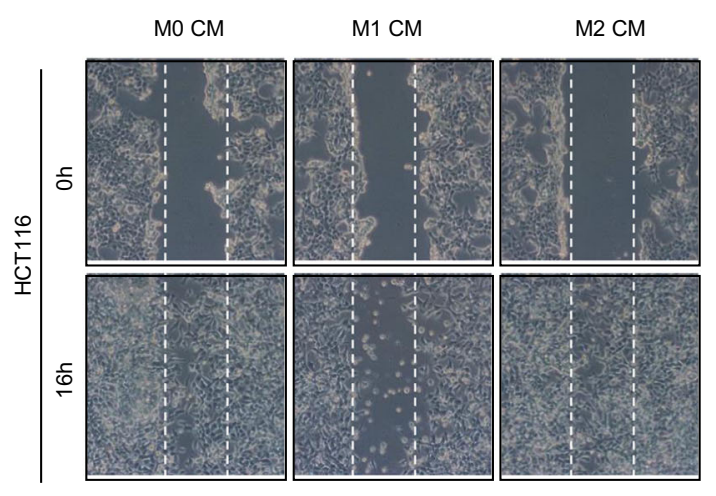

E
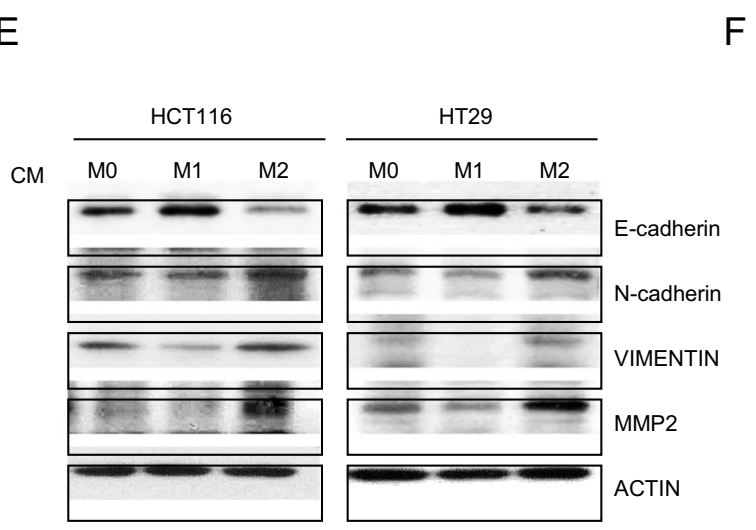

F

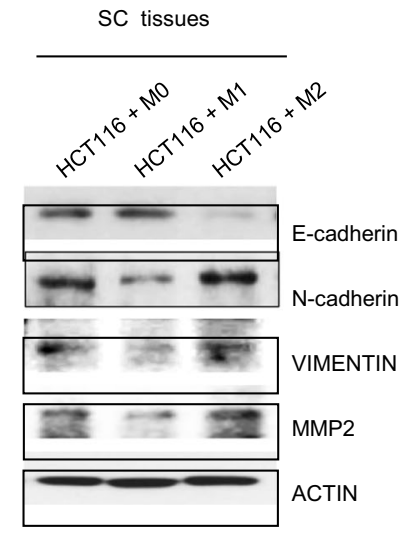

Fig. 3 Effects of macrophage CM on EMT in colon cancer cells. a Transwell Matrigel invasion assays of HT29 and HCT116 cells after coculture with macrophage CM for 24 h. b HT29 cells were subjected to immunofluorescence staining with an antibody against E-cadherin, were mounted with DAPI-containing mounting solution and observed at $\times 200$ and $\times 400$ magnification. A wound-healing assay was performed by creating a wound on a confluent monolayer of HT29 c and HCT116 d cells during incubation with macrophage CM for $16 \mathrm{~h}$. Expression levels of EMT markers in HCT116 and HT29 cells treated with macrophage CM for $24 \mathrm{~h}$ e and in mouse subcutaneous tissues $\mathbf{f}$ were evaluated by western blotting. Actin was used as a loading control.

Ex vivo experiments confirm the role of $\mathrm{M} 1$ and $\mathrm{M} 2$ macrophages in colon cancer cell pathology

To further confirm the data obtained above, ex vivo analysis was performed using PDCs (Supplementary Fig. 1E). M1 CM significantly decreased the viability of PDCs, whereas M2 CM significantly increased the viability of PDCs compared to that of MO CM treatment (Fig. 5a). To determine whether cell survival was associated with macrophage CM, western blotting was performed using antibodies against representative apoptosis markers. As 


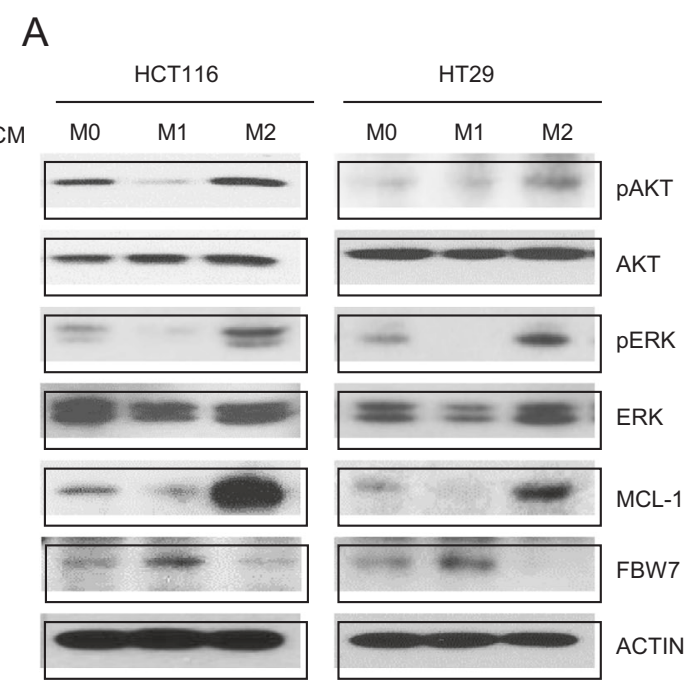

C

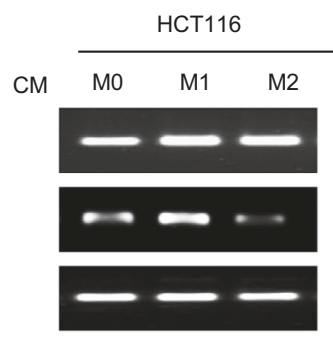

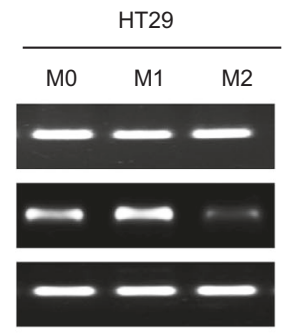

$M C L-1$

FBXW7

GAPDH
B

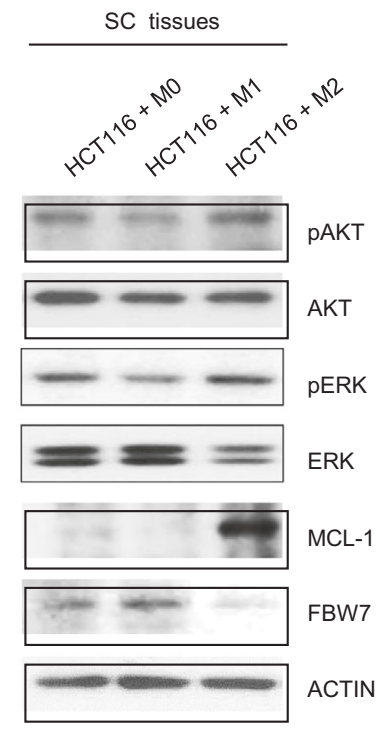

D

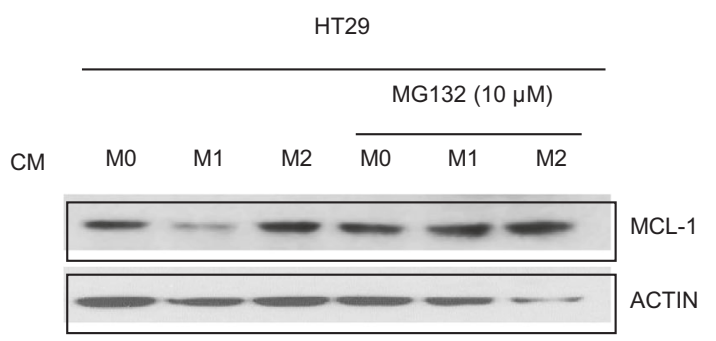

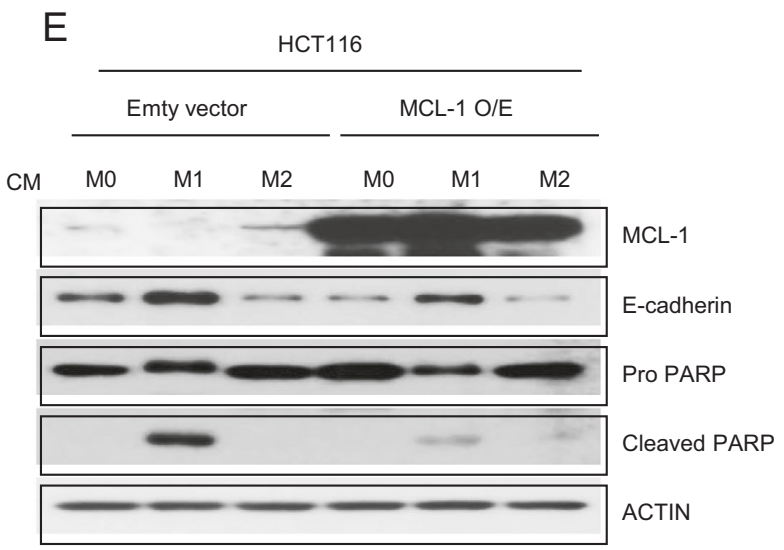

Fig. 4 Opposite effects of M1 macrophage CM and M2 macrophage CM on FBW7-mediated MCL-1 degradation of colon cancer cells. Expression levels of AKT, ERK, MCL-1, and FBW7 in a macrophage CM-treated HCT116 and HT29 cells and in $\mathbf{b}$ mouse subcutaneous tissues transplanted with long-term coculture and differentiated M0, M1, and M2 macrophages. c mRNA levels of MCL-1 and FBW7 were evaluated by RT-PCR after macrophage CM treatment for $24 \mathrm{~h}$. $\mathbf{d}$ HT29 cells were exposed to MG132 for $1 \mathrm{~h}$ and incubated with macrophage CM for $24 \mathrm{~h}$. e HCT116 cells were transfected with either empty vector or MCL-1 expression vector for $24 \mathrm{~h}$, followed by incubation with macrophage CM for another $24 \mathrm{~h}$. Actin was used as a loading control.

expected, M1 CM-induced cell death through apoptosis, whereas M2 CM enhanced survival of PDCs (Fig. 5b). The effect of macrophage CM on the EMT features of PDCs was also determined using the same respective cell lysates. The expression levels of the well-known EMT markers E-cadherin, N-cadherin, VIMENTIN, and MMP2 in M0, M1, or 

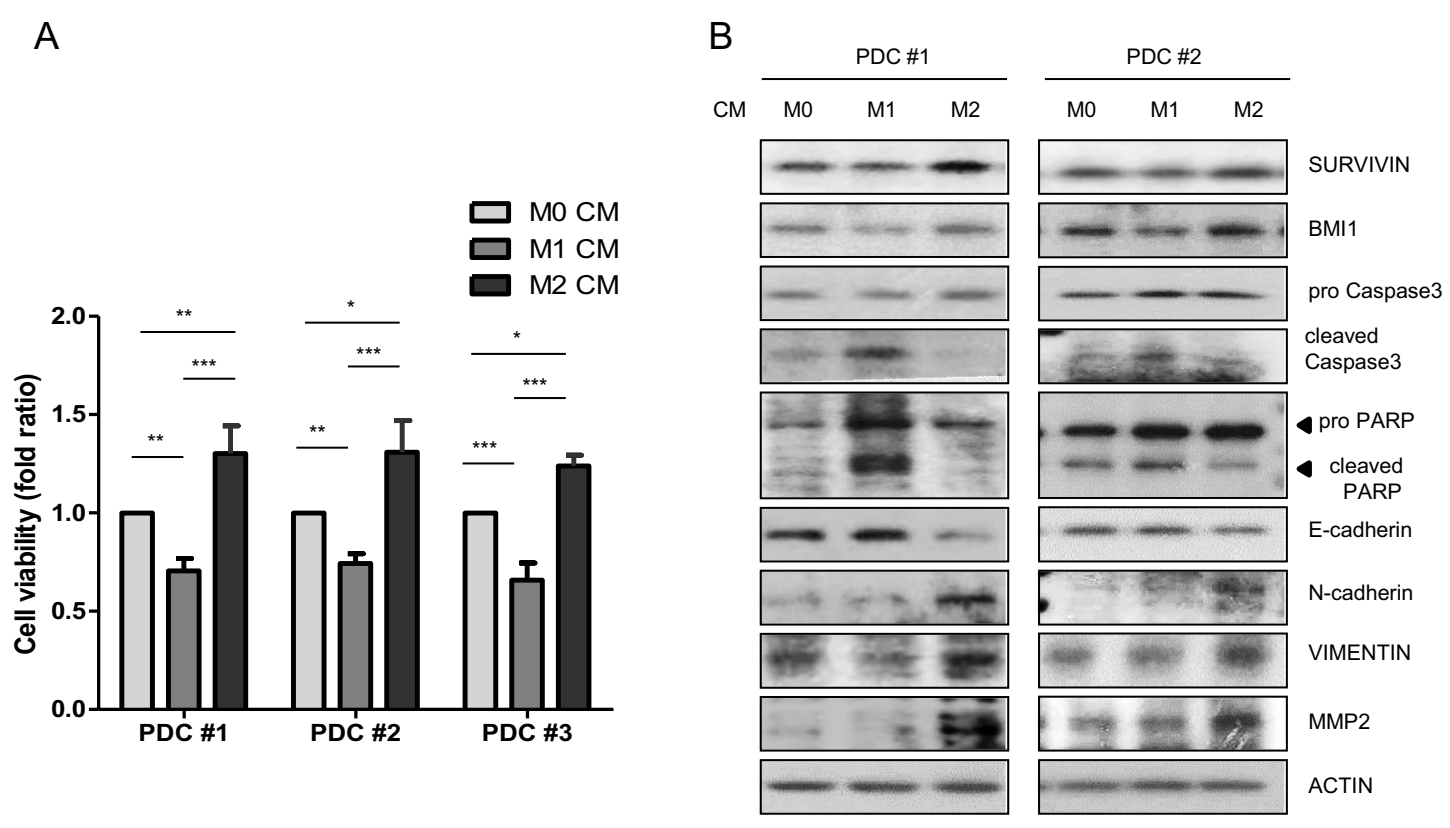

C

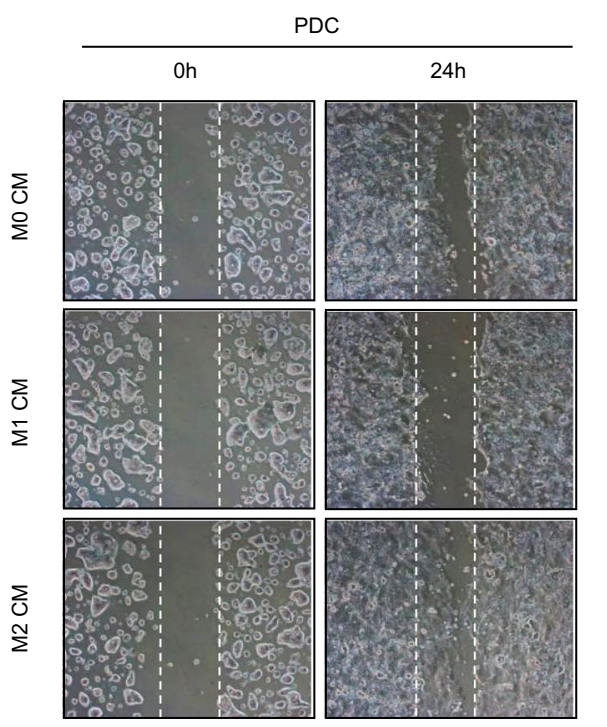

$\mathrm{D}$

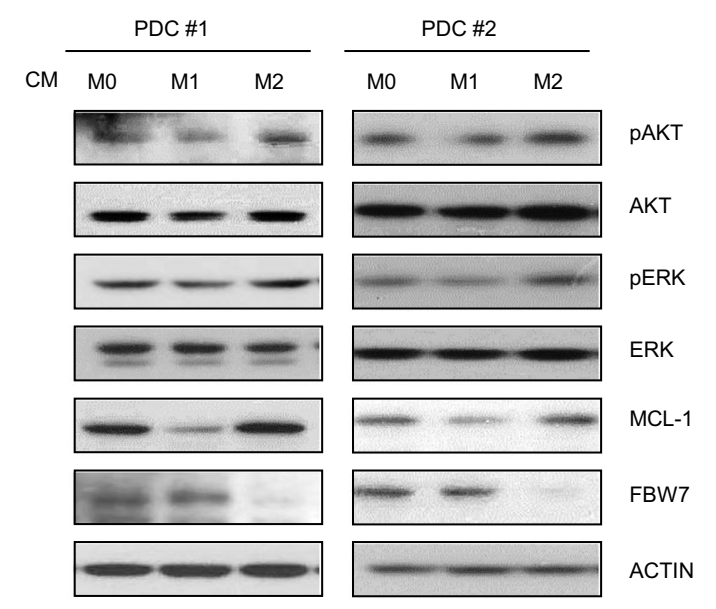

Fig. 5 Ex vivo analysis of the role of macrophage CM in colon cancer patient-derived cells (PDCs). a Estimation of PDC viability after coculture with macrophage CM for $24 \mathrm{~h}$ using the WST-1 assay. Error bars are derived from three independent experiments. $\mathbf{b}$ Expression levels of apoptosis and EMT markers in macrophage CM-treated PDCs were measured by western blotting. $\mathbf{c}$ Wound-healing ability was evaluated by creating wounds on a confluent monolayer of PDCs using 1-Dish 35-mm-high culture inserts. $\mathbf{d}$ Activation of AKT and ERK and expression levels of MCL-1 and FBW7 assessed by western blotting. Actin was used as a loading control. The results are presented as the mean \pm SE. ${ }^{* *} P<0.01$; ${ }^{* * *} P<0.001$.

M2 CM-treated PDCs were also determined. The results confirmed that M2 macrophages enhanced EMT progression (Fig. 5b). The representative image shown in Fig. 5c revealed that M1 CM inhibited the migration of PDCs, whereas M2 CM-induced migration of PDCs compared with that of MO CM. Consistent with our previous results (Fig. 4) showing that posttranslational modulation of the FBW7-Mcl-1 axis was an underlying mechanism involved in macrophage-mediated colon cancer cell proliferation, the involvement of the FBW7-Mcl-1 axis in PDCs was also found in CM-treated macrophages (Fig. 5d).

\section{Effects of macrophage PI3K $\gamma$ inhibition on colon cancer cell viability and EMT status}

Although our results confirmed the effect of macrophage $\mathrm{CM}$ on colon cancer cell survival and EMT 
phenotypes, target macrophage molecules need to be determined to apply these findings to clinical immunotherapy. PI3K $\gamma$ is specifically expressed in myeloid cells but not in cancer cells. PI3Ky enhances myeloid cell trafficking during the inflammatory response and carcinogenesis ${ }^{36}$. Hence, PI3K $\gamma$ was a focus of this study as a promising candidate target for colon cancer immune therapy. As small-molecule inhibitors might be more useful for clinical approaches than genetic modulation, the biological effects of the PI3K $\gamma$ inhibitor TG100-115 (Selleckchem) were determined. RT-PCR performed in triplicate revealed that proinflammatory cytokines (upper panel) were upregulated in M1 macrophages, whereas antiinflammatory cytokines (lower panel) were upregulated in M2 macrophages (Supplementary Fig. 1C). TG100-115-induced PI3K $\gamma$ inhibition significantly stimulated proinflammatory cytokine expression and M1 macrophage marker expression but reduced antiinflammatory cytokine expression and M2 macrophage marker expression in both M1 and M2-differentiated macrophages (Fig. 6a, b, Supplementary Fig. 2C). Similar results were observed when M1 and M2 macrophages were treated with another well-known PI3K $\gamma$ inhibitor, IPI-549 (Supplementary Fig. 3C). In other words, PI3K $\gamma$ controls macrophage switching between immune tolerance and immune surveillance by regulating cytokines. Next, CM was collected from TG100-115-treated M0, M1, or M2 macrophages. As expected, M0, M1, and M2 CM collected from TG100-115-treated macrophages significantly reduced the viability of HT29 cells (Fig. 6c) and PDCs (Supplementary Fig. 2D) in a dose-dependent manner regardless of macrophage subtype. These results suggest that blocking $\mathrm{PI} 3 \mathrm{~K} \gamma$ in macrophages promotes cytotoxic effects in colon cancer cells. Subsequently, the effect of PI3K $\gamma$ inhibition in macrophages on EMT and the apoptosis-related death of colon cancer cells was examined by western blotting. Our results showed that M0, M1, and M2 CM from TG100-115-treated macrophages restored the protein levels of E-cadherin (an EMT marker) as well as the apoptosis markers cleaved PARP and SURVIVIN in HT29 cells regardless of macrophage subtype (Fig. 6d). Furthermore, CM from TG100-115treated macrophages at the tested concentrations reduced MCL-1 protein expression but enhanced FBW7 expression in both HT29 cells (Fig. 6e) and PDCs (Fig. 6f) regardless of macrophage subtype. The WST-1 assay showed that TG100-115 had no effect on the viability of M1 macrophages, whereas TG100-115 significantly decreased the viability of M2 macrophages in a dosedependent manner (Supplementary Fig. 2E). Collectively, these data indicate that PI3K $\gamma$ targeting in TAMs could switch macrophage-derived cytokines, thus affecting colon cancer cell growth and EMT features.

\section{Inhibition of PI3Ky attenuates tumor growth in xenograft models associated with infiltrated macrophages}

To verify our results, we used a syngeneic mouse model of BALB/c wild-type mice and the CT26 mouse colon cancer cell line. In brief, $5 \times 10^{5}$ cells were subcutaneously injected into the mouse flank, and TG100-115 was used for treatment every other day via intraperitoneal injection (Fig. 7a). The mice treated with the PI $3 \mathrm{~K} \gamma$ antagonist TG100-115 showed dramatically suppressed tumor growth in the CT26 xenograft model (Fig. 7b). To observe macrophage infiltration in each tissue, we dissociated tumor cells and performed cytometry analysis using specific markers for total macrophages $\left(\mathrm{CD} 11 \mathrm{~b}^{+} \mathrm{F} 4 / 80^{+}\right)$. We found that PI3K $\gamma$ inhibition induced macrophage infiltration into the tumor region (Fig. 7c left panel). Blocking PI3K $\gamma$ activity with TG100-115 treatment increased the proportion of M1-like macrophages $\left(\mathrm{CD} 11 \mathrm{~b}^{+} \mathrm{F} 4 /\right.$

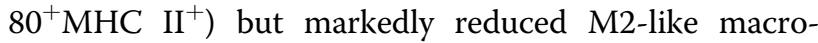
phages $\left(\mathrm{CD} 11 \mathrm{~b}^{+} \mathrm{F} 4 / 80^{+} \mathrm{CD} 206^{+}\right.$) (Fig. $7 \mathrm{c}$ right panel, Fig. 7d). Relative mRNA expression levels of M1-like proinflammatory cytokines (il-1 $\beta$ and $c x c l 10$ ) were upregulated, whereas those of M2-like antiinflammatory cytokines (il-10 and tgf- $\beta$ ) were downregulated in TG100115-treated tumors (Fig. 7e). Moreover, the expression levels of survival markers and EMT markers in TG100115-treated mouse tissues confirmed that PI3Ky inhibition reversed tumor progression through the FBW7MCL-1 axis (Fig. 7f).

\section{Association between M1 and M2 macrophage infiltration and prognostic importance}

The major M1 macrophage biomarker CD86 and M2 macrophage biomarker $\mathrm{CD} 163^{37}$ were used to identify TAM phenotypes. To test whether CD86 and CD163 could be used to distinguish different subpopulations of M1 or M2 macrophages, CRC tissues were analyzed by multiplex staining using the Opal Kit (see Materials and Methods). The results showed that CD86 and CD163 were expressed in different populations of macrophages. The amounts of M2 $\left(\mathrm{CD} 163^{+}\right)$and $\mathrm{M} 1\left(\mathrm{CD} 86^{+}\right)$macrophages showed individual variation in the tumor microenvironment (Fig. 8a). To determine the prognostic impact of M1 or M2 macrophage infiltration in the tumor microenvironment, survival outcomes were analyzed with Kaplan-Meier analysis among a total of 232 CRC patients (stage I, $n=32$; stage II, $n=70$; stage III, $n=76$; and stage IV, $n=54$ ). Based on the results of the M1 and M2 macrophage markers, these 232 cases were divided into the following two groups: (1) M2/M1 $<3$ and (2) M2/ $\mathrm{M} 1>3$. The results revealed that patients with $\mathrm{M} 2 / \mathrm{M} 1<3$ had significantly better PFS ( $p=0.033$, Fig. 8b, left panel) and overall survival $(p=0.043$, Fig. $8 \mathrm{~b}$, right panel) than patients with $\mathrm{M} 2 / \mathrm{M} 1>3$. 
A

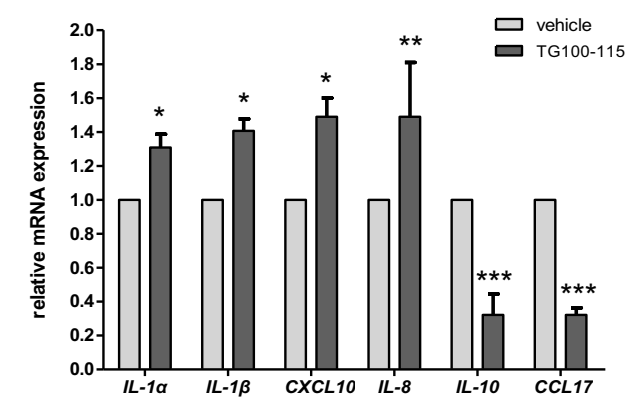

C

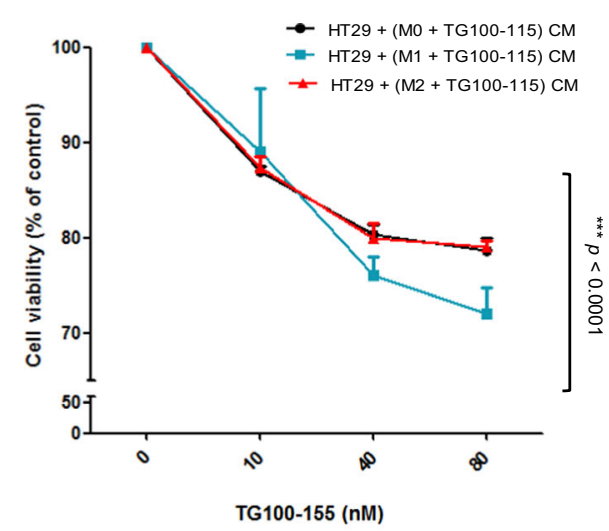

E

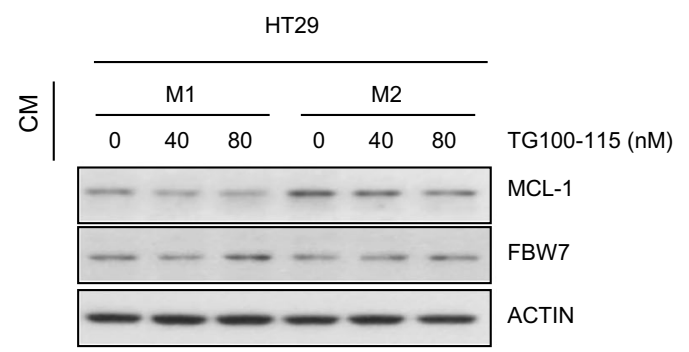

B

M2 macrophage

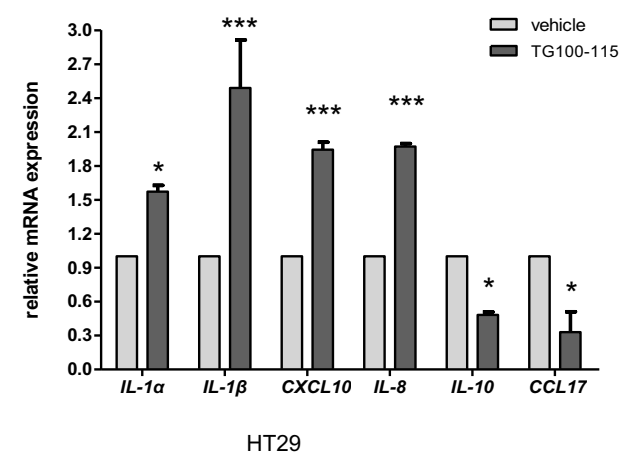

D

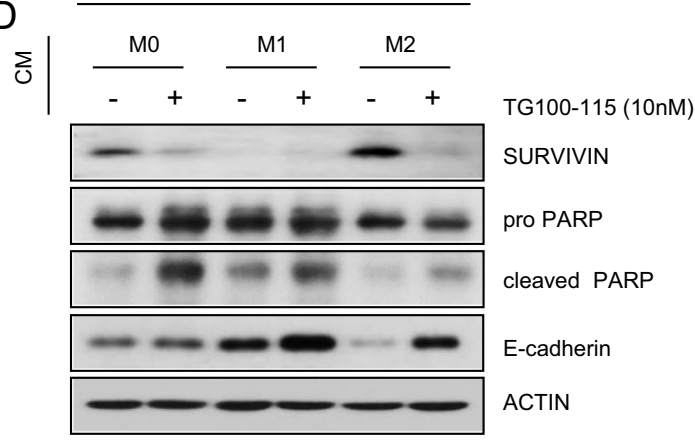

$\mathrm{F}$

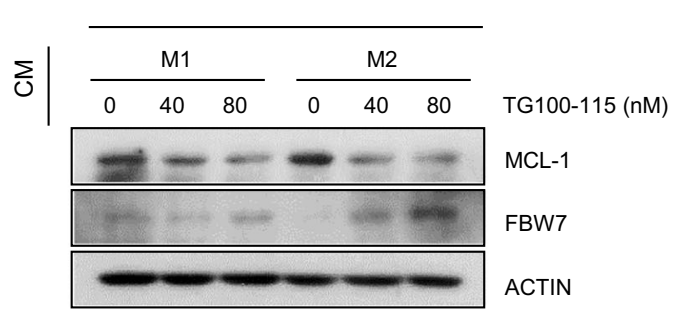

Fig. 6 Role of PI3Ky in TAM-mediated colon cancer cell viability and EMT characteristics. mRNA expression levels of genes involved in the proinflammatory response (IL-1a, IL-1 $\beta, C X C L 10$, and IL-8) and antiinflammatory response (IL-10 and CCL17) were evaluated by real-time PCR in M1 $\mathbf{a}$ and M2 b macrophages with or without treatment with $10 \mathrm{~nm}$ TG100-115 (PI3Ky inhibitor) for $18 \mathrm{~h}$. c HT29 cells were exposed to TG100-115-treated macrophage CM for $24 \mathrm{~h}$. Cell viability was then measured by WST-1 assay. $\mathbf{d}$ Expression levels of apoptosis- and EMT-related markers in CM-treated HT29 cells. CMs were derived from TG100-115 (10 nM)-treated or untreated M0, M1, and M2 macrophages. HT29 cells e and PDCs $\mathbf{f}$ were incubated with TG100-115-treated macrophage CM for $24 \mathrm{~h}$. Protein levels of MCL-1 and FBW7 were evaluated using western blotting. Actin was used as a loading control. The results are presented as the mean \pm SE. ${ }^{*} P<0.05 ;{ }^{* *} P<0.01 ;{ }^{* *} P<0.001$.

\section{Discussion}

Multiple studies have shown that TAMs are associated with cancer progression in many types of malignancies, including liver, breast, gastric, and lung cancers ${ }^{8,38,39}$. Although these studies have implicated infiltrated TAM density and polarization status in 5-year survival outcomes, the impact of the ratio of infiltrated TAMs on survival in CRC remains controversial ${ }^{16,40}$. Moreover, mechanistic studies of TAM-related tumor progression and potential immunotherapeutic targets in TAMs have been sparse. To the best of our knowledge, this is the first attempt to estimate the mechanistic role of M1/M2 TAM subtypes in MCL-1-mediated cancer cell proliferation and the EMT process using in vivo, in vitro, and ex vivo approaches. In addition, the effects of inhibiting macrophage PI3K $\gamma$ on tumor suppression were examined in this study. Our results could help validate the immunotherapeutic relevance of targeting $\mathrm{PI} 3 \mathrm{~K} \gamma$ in $\mathrm{CRC}$ treatment. 


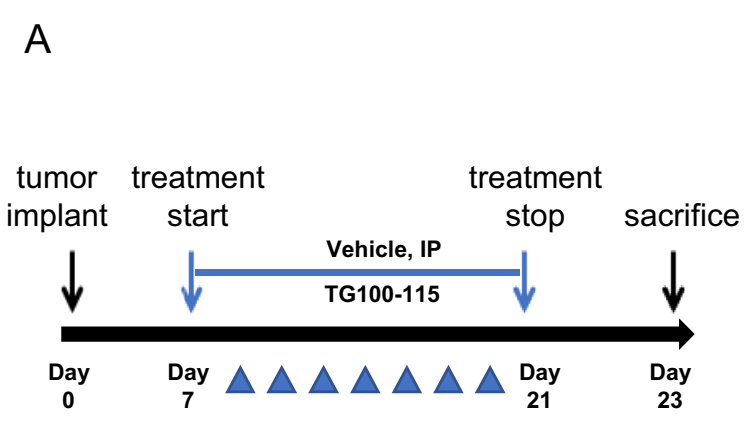

C
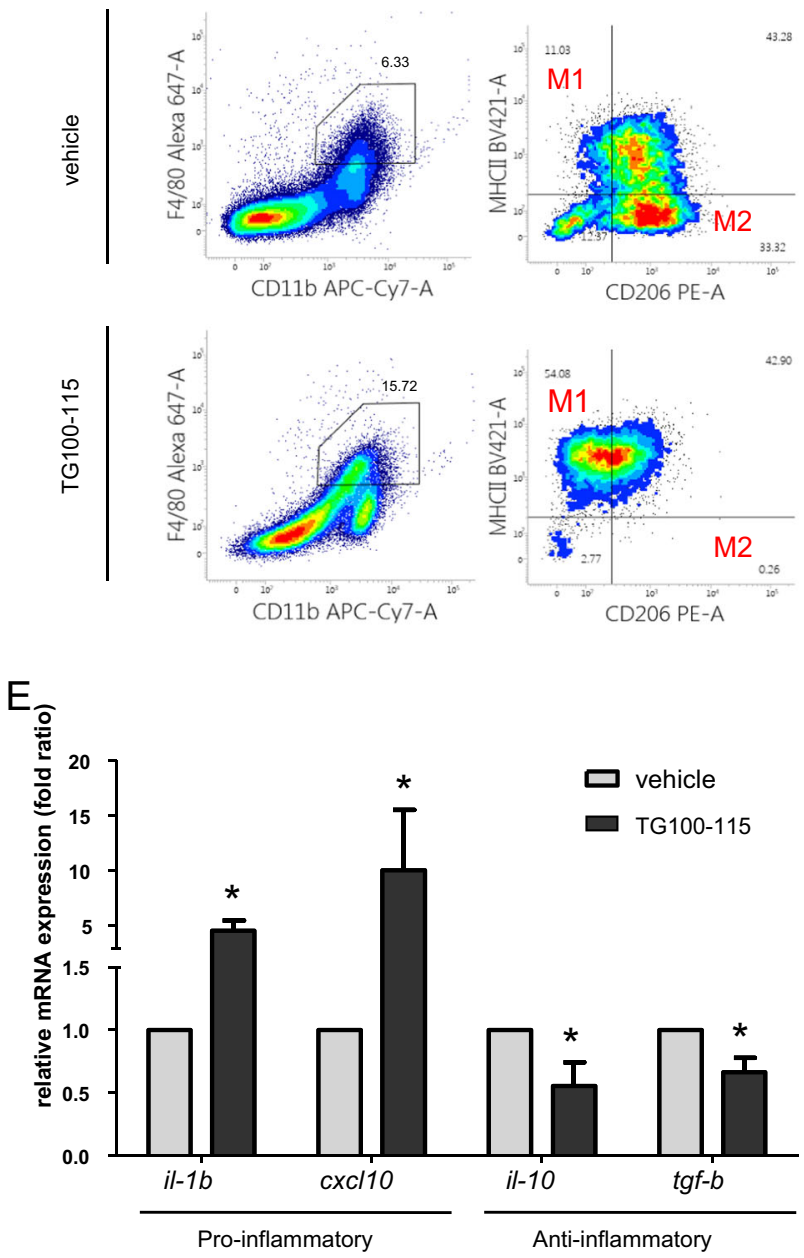

B
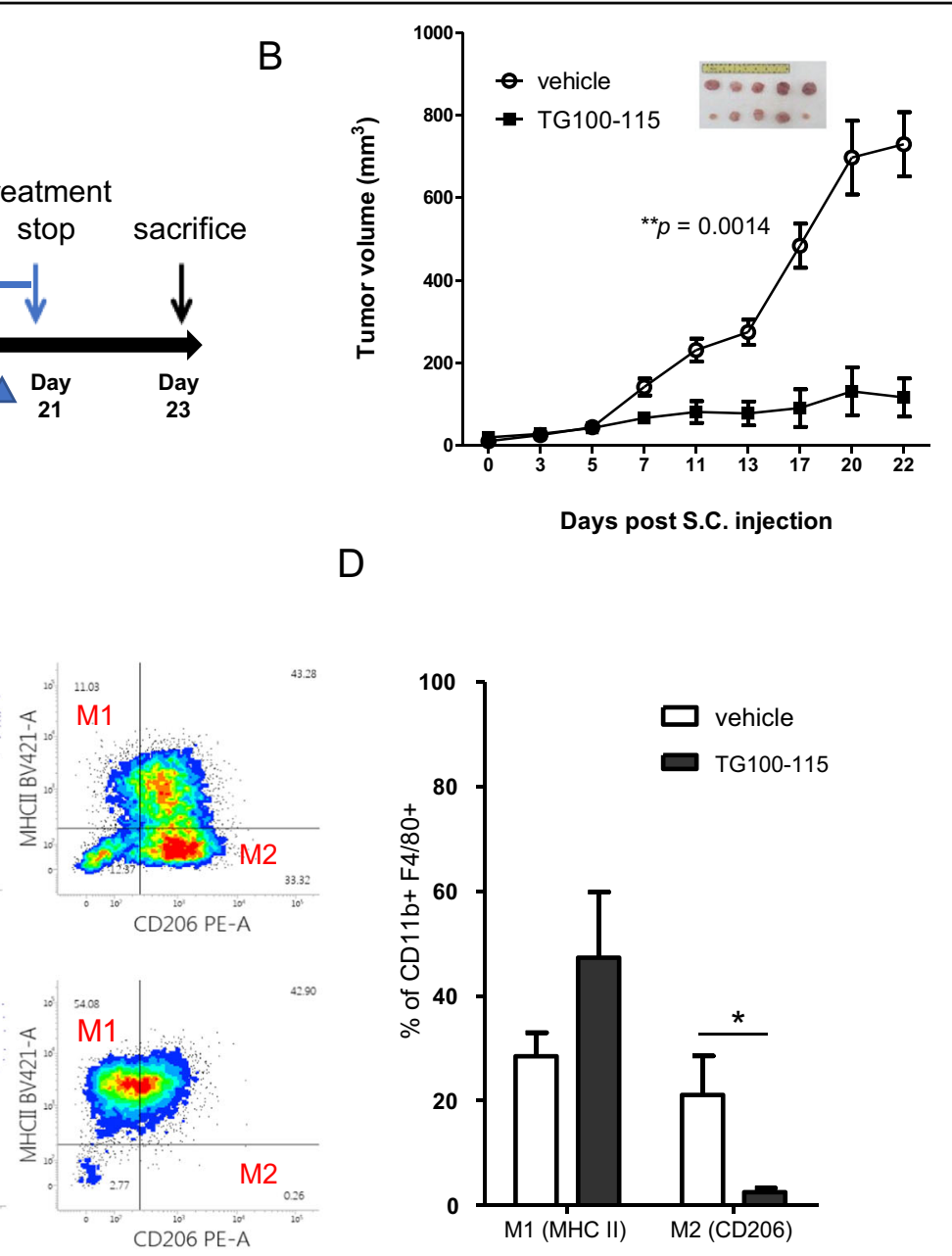

$\mathrm{F}$

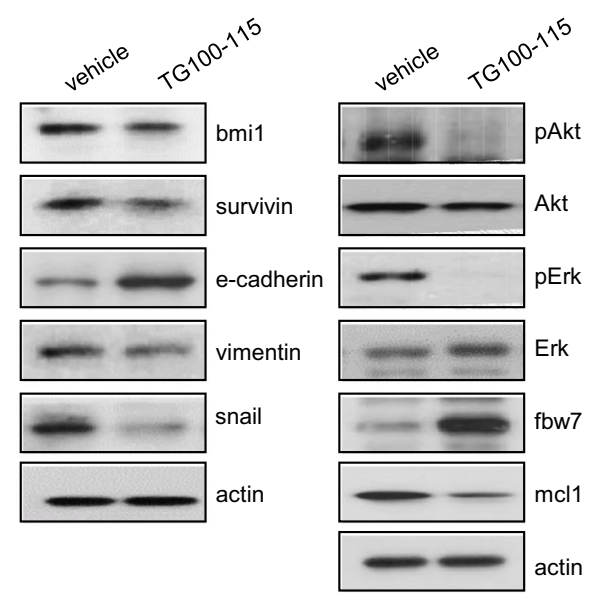

Fig. 7 Inhibition of PI3Ky attenuates tumor growth in xenograft models associated with infiltrated macrophages. a Experimental procedure illustrating the TG100-115 treatment regimen in BALB/c mice. b CT26 cells ( $5 \times 10^{5}$ cells/mouse) were subcutaneously injected into the flanks of 6 week-old mice. Mean tumor volume of subcutaneously implanted vehicle- or TG100-115-treated mice $(n=5)$ and representative images of subcutaneous tumors at day 16 after treatment with vehicle or TG100-115 (box) are shown. c FACS analysis and quantification of CD11 b $\mathrm{b}^{+}$F/80 ${ }^{+}$ (TAM) cell populations in CT26 tumors at day 14 posttreatment $(n=5)$ and expression levels of MHCII (M1) and CD206 (M2) in CD11 b ${ }^{+}$F4/80 $0^{+}$cell populations. $\mathbf{d}$ Graph showing the percentage of each population (M1, M2) in the vehicle-treated group in comparison with the TG100-115-treated group. e mRNA expression levels of genes involved in the proinflammatory response (il- $1 \beta, c x c / 10$ ) and antiinflammatory response (il-10 and tgf- $\beta$ ) were evaluated by real-time PCR in vehicle and TG100-155-treated groups. f Representative western blot analysis showing survival/EMT-related protein expression as well as ERK/AKT-FBW7-MCL-1 signal axis regulation in vehicle- and TG100-155-treated mouse tumor tissues. 
A
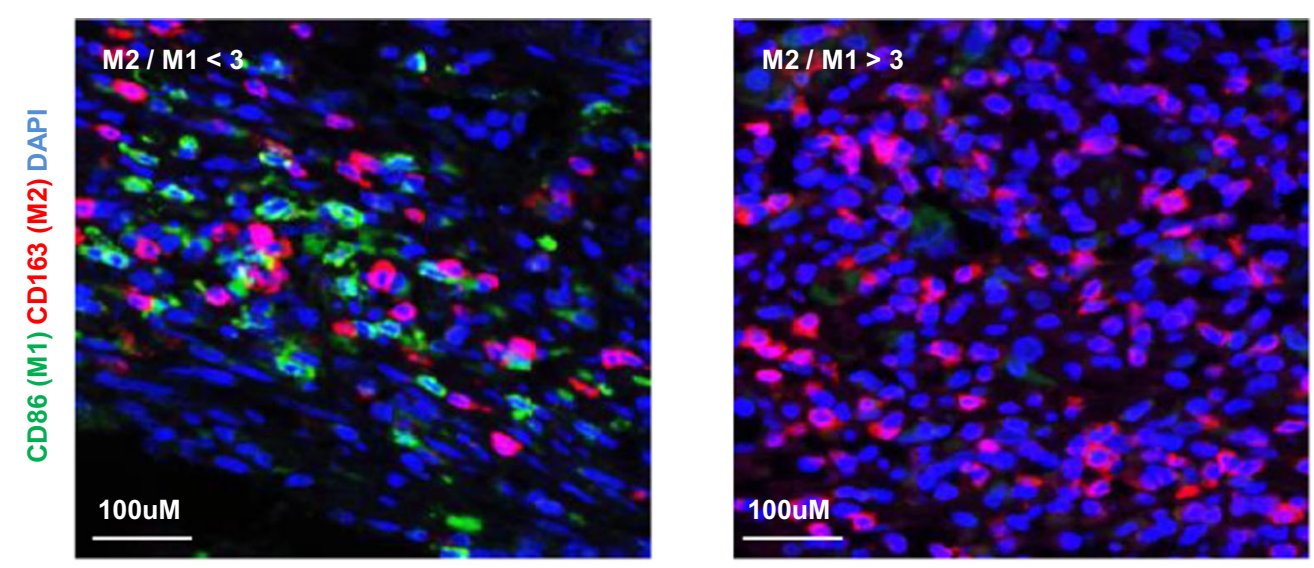

B
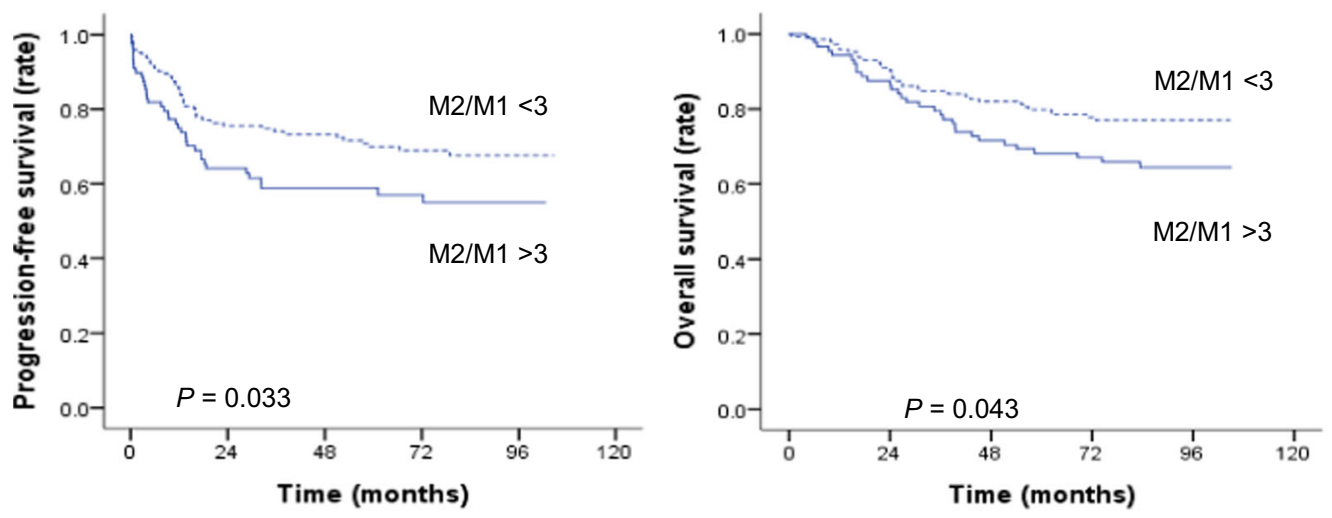

Fig. 8 Survival analysis according to the M2 marker (CD163) to M1 marker (CD86) ratio in the validation cohort. a Representative immunohistochemical staining for markers of M1 macrophages (CD86, green) and M2 macrophages (CD163, red) in CRC tissues. Dried slides were scanned using the PerkinElmer Vectra 3.0 platform at $\times 20$ magnification. Nuclei were shown by DAPI staining (blue). Scale bar: $100 \mu \mathrm{m}$.

b Kaplan-Meier curves for progression-free survival (left panel) and overall survival (right panel) of the two groups were divided by an optimal cutoff value of 3 for the ratio of M2 marker (CD163) to M1 marker (CD86). Statistical analysis by long-rank test: $p=0.033$ and $p=0.043$, respectively.

The results of the clinical data analysis of 232 CRC patients revealed that the ratio of M2 to M1 macrophages was associated with the survival outcomes of CRC patients (Fig. 8). Our results clearly showed that PFS and overall survival were significantly prolonged in patients with an M2 to M1 macrophage ratio < 3 compared with those with a ratio larger than 3 . In contrast to our results, Nagorsen et al. ${ }^{41}$ reported that M2 macrophage infiltration in CRC is correlated with better survival outcomes. However, their study did not estimate the parallel presence of M1 macrophage infiltration. The distribution of M1 and M2 macrophages and their ratio in CRC are believed to be very important in developing comprehensive immunotherapeutic strategies. Similar to our results, Edin et al. ${ }^{16}$ found a positive correlation between infiltrated M1 macrophages and survival outcomes in CRC patients. However, unlike our results, they concluded that M2 macrophage infiltration was increased concomitantly with M1 macrophage infiltration. The unique value of our clinical analysis compared to previous reports was that we revealed that the M2/M1 ratio could predict better prognostic outcomes. Both in vitro and in vivo studies demonstrated that M1 and M2 macrophages had opposite effects on the proliferation and EMT phenotypes of colon cancer cells. Data from colon cancer cell lines and a mouse xenograft model consistently showed that the AKT and ERK pathways were associated with FBW7-related MCL-1 degradation in M1 or M2 cocultured colon cancer cells. To the best of our knowledge, this is the first study to use an ex vivo approach with PDCs to confirm our in vitro and in vivo data. PDCs are generated by directly resecting patient tumors into single cells. Such models can replicate the features of primary patient tumors. This can help overcome potential barriers of in vitro models ${ }^{42}$. Hence, our study went one step further than existing reports on the 
role of TAMs in the colon cancer tumor microenvironment. Intracellular PI3K $\gamma$ has recently been suggested to be a target for immunotherapy in macrophages. PI3K $\gamma$ inhibition can switch macrophage phenotypes from immune tolerance to immune surveillance by regulating the NFKB and mTOR-C/EBP pathways ${ }^{26,43}$. In agreement with previous reports, our results revealed that $\mathrm{PI} 3 \mathrm{~K} \gamma$ inhibition increased antitumor cytokines and decreased protumor cytokines in macrophages, thus enhancing apoptosis-related cancer cell death regardless of the TAM subtype. Moreover, apoptosis marker and EMT marker expression, as well as the FBW7-MCL-1 axis, were reversed via CM obtained from TG110-115-treated M1 and M2 macrophages. Furthermore, PI3K $\gamma$ inhibition demonstrated potential therapeutic use in an in vivo xenograft model, as regression was seen in $80 \%$ of syngeneic mouse tumors after treatment with TG100-115. We found that PI3K $\gamma$ inhibition increased the number of tumor-infiltrated macrophages and switched their polarization from immunosuppressive M2 macrophages to more proinflammatory M1 macrophages. Furthermore, we confirmed that tumor regression was related to FBW7-MCL-1 signaling associated with the polarization of infiltrated macrophages. Moreover, TG100-115 significantly decreased the viability of M2 macrophages without significantly affecting the viability of M1 macrophages. These results indicate that $\mathrm{PI} 3 \mathrm{~K} \gamma$ in macrophages might be a promising target in the development of immunotherapy for colon cancer.

Since one study reported a $0 \%$ response rate of colon cancer MSS patients to pembrolizumab in $2015^{44}$, the results of PD-1/PD-L1 antibody treatment for MSS tumor types have not been established. Immune checkpoint inhibitor treatment for colon cancer patients has only been established in MSI patients, who represent $<15 \%$ of colon cancer patients ${ }^{45,46}$. MSI patients exhibit microsatelliteunstable disease types that are characterized as mismatch repair deficient. Unlike the MSS tumor type, which is a mismatch repair proficient disease type, MSI tumors contain many mutations that create unique new antigens. Hence, active immunotherapy strategies that involve macrophage targeting for MSS tumor types are promising. Hence, the results we present here provide a rationale for targeting macrophages by reprogramming macrophage characteristics from protumoral to antitumoral, which may affect the colon tumor microenvironment from cold tumors to hot tumors.

In summary, the correlation between the M1 to M2 ratio and survival outcomes and the underlying signaling pathway involved were examined by using in vitro, in vivo, and ex vivo approaches in this study. Our results demonstrated that the cancer cell progression induced by M2 macrophages was mechanistically linked to FBW7mediated MCL-1 stabilization in colon cancer cells.
Moreover, inhibition of endogenous PI3Ky in M2 macrophages could switch macrophage function from "protumor" to "antitumor", thus affecting colon cancer cell survival in the tumor microenvironment. Although further improvements are required to clarify the internal signaling pathway of PI3K $\gamma$ in TAMs, our results identified FBW7-MCL-1 as a key axis in M2 macrophagerelated colon cancer cell progression.

\section{Acknowledgements \\ This research was supported by grants (NRF-2016R1A6A3A11933598, 2015R1A2A2A01003225, and NRF-2020R1A2C2006408) from the Basic Science Research Program through the National Research Foundation of Korea (NRF) funded by the Ministry of Education, Science, and Technology. This study was also supported by a grant (OTC1190661) funded by Samsung Medical Center, Korea.}

\section{Author details}

${ }^{1}$ Sungkyunkwan University School of Medicine, Seoul, Republic of Korea. ${ }^{2}$ Institute for Future Medicine Samsung Medical Center, Seoul, Republic of Korea. ${ }^{3}$ Department of Colorectal Surgery, Hallym University Sacred Heart Hospital, Hallym University College of Medicine, Anyang, Korea. ${ }^{4}$ Department of Surgery, Samsung Medical Center, Sungkyunkwan University School of Medicine, Seoul, Republic of Korea. ${ }^{5}$ Department of Health Sciences and Technology, Samsung Advanced Institute for Health Sciences and Technology, Sungkyunkwan University, Seoul, Republic of Korea

\section{Conflict of interest}

The authors declare that they have no conflict of interest.

\section{Publisher's note}

Springer Nature remains neutral with regard to jurisdictional claims in published maps and institutional affiliations.

Supplementary information accompanies this paper at https://doi.org/ 10.1038/s12276-020-0436-7.

Received: 25 June 2019 Revised: 23 February 2020 Accepted: 8 April 2020. Published online: 22 May 2020

\section{References}

1. Murdoch, C., Muthana, M., Coffelt, S. B. \& Lewis, C. E. The role of myeloid cells in the promotion of tumour angiogenesis. Nat. Rev. Cancer $\mathbf{8}$, 618-631 (2008).

2. Peddareddigari, V. G., Wang, D. \& Dubois, R. N. The tumor microenvironment in colorectal carcinogenesis. Cancer Microenviron. 3, 149-166 (2010).

3. Gajewski, T. F., Schreiber, H. \& Fu, Y. X. Innate and adaptive immune cells in the tumor microenvironment. Nat. Immunol. 14, 1014-1022 (2013).

4. Pollard, J. W. Tumour-educated macrophages promote tumour progression and metastasis. Nat. Rev. Cancer 4, 71-78 (2004).

5. Ostuni, R., Kratochvill, F., Murray, P. J. \& Natoli, G. Macrophages and cancer: from mechanisms to therapeutic implications. Trends Immunol. 36, 229-239 (2015).

6. Mantovani, A. \& Allavena, P. The interaction of anticancer therapies with tumor-associated macrophages. J. Exp. Med. 212, 435-445 (2015).

7. Stossi, F., Madak-Erdogan, Z. \& Katzenellenbogen, B. S. Macrophage-elicited loss of estrogen receptor-alpha in breast cancer cells via involvement of MAPK and c-Jun at the ESR1 genomic locus. Oncogene 31, 1825-1834 (2012).

8. Yuan, A. et al. Opposite effects of M1 and M2 macrophage subtypes on lung cancer progression. Sci. Rep. 5, 14273 (2015).

9. Chai, C. Y. et al. Hypoxia-inducible factor-1alpha expression correlates with focal macrophage infiltration, angiogenesis and unfavourable prognosis in urothelial carcinoma. J. Clin. Pathol. 61, 658-664 (2008).

10. Zhang, M. et al. A high M1/M2 ratio of tumor-associated macrophages is associated with extended survival in ovarian cancer patients. J. Ovarian Res. 7, 19 (2014). 
11. Koukourakis, M. I. et al. Different patterns of stromal and cancer cell thymidine phosphorylase reactivity in non-small-cell lung cancer: impact on tumour neoangiogenesis and survival. Br. J. Cancer 77, 1696-1703 (1998).

12. Shimura, S. et al. Reduced infiltration of tumor-associated macrophages in human prostate cancer: association with cancer progression. Cancer Res. 60, 5857-5861 (2000).

13. Migita, T. et al. Differing expression of MMPs-1 and -9 and urokinase receptor between diffuse- and intestinal-type gastric carcinoma. Int. J. Cancer 84, 74-79 (1999).

14. Nakayama, Y. et al. Relationships between tumor-associated macrophages and clinicopathological factors in patients with colorectal cancer. Anticancer Res. 22, 4291-4296 (2002).

15. Klintrup, K. et al. Inflammation and prognosis in colorectal cancer. Eur. J. Cancer 41, 2645-2654 (2005).

16. Edin, S. et al. The distribution of macrophages with a M1 or M2 phenotype in relation to prognosis and the molecular characteristics of colorectal cancer. PloS ONE 7, e47045 (2012).

17. Gulubova, M. et al. The density of macrophages in colorectal cancer is inversely correlated to TGF-beta1 expression and patients' survival. J. Mol. Histol. 44, 679-692 (2013).

18. Koelzer, V. H. et al. Phenotyping of tumor-associated macrophages in colorectal cancer: impact on single cell invasion (tumor budding) and clinicopathological outcome. Oncoimmunology 5, e1106677 (2016).

19. Cavnar, M. J. et al. Tumor-associated macrophage infiltration in colorectal cancer liver metastases is associated with better outcome. Ann. Surg. Oncol. 24, 1835-1842 (2017)

20. Zhou, Q. et al. The density of macrophages in the invasive front is inversely correlated to liver metastasis in colon cancer. J. Transl. Med. 8, 13 (2010).

21. Vanhaesebroeck, B., Stephens, L. \& Hawkins, P. PI3K signalling: the path to discovery and understanding. Nat. Rev. Mol. Cell Biol. 13, 195-203 (2012).

22. Martin, E. L. et al. Phosphoinositide-3 kinase gamma activity contributes to sepsis and organ damage by altering neutrophil recruitment. Am. J. Respir. Crit. Care Med. 182, 762-773 (2010).

23. Byles, V. et al. The TSC-mTOR pathway regulates macrophage polarization. Nat. Commun. 4, 2834 (2013)

24. Chaurasia, B. et al. Phosphoinositide-dependent kinase 1 provides negative feedback inhibition to Toll-like receptor-mediated NF-kappaB activation in macrophages. Mol. Cell Biol. 30, 4354-4366 (2010).

25. Arranz, A. et al. Akt1 and Akt2 protein kinases differentially contribute to macrophage polarization. Proc. Natl. Acad. Sci. USA 109, 9517-9522 (2012).

26. Kaneda, M. M. et al. PI3Kgamma is a molecular switch that controls immune suppression. Nature 539, 437-442 (2016).

27. Kaneda, M. M. et al. Macrophage PI3Kgamma drives pancreatic ductal adenocarcinoma progression. Cancer Discov. 6, 870-885 (2016).

28. Gao, L. et al. Tumor associated macrophages induce epithelial to mesenchymal transition via the EGFR/ERK1/2 pathway in head and neck squamous cell carcinoma. Oncol. Rep. 40, 2558-2572 (2018).
29. Genin, M., Clement, F., Fattaccioli, A., Raes, M. \& Michiels, C. M1 and M2 macrophages derived from THP-1 cells differentially modulate the response of cancer cells to etoposide. BMC Cancer 15, 577 (2015).

30. Canel, M., Serrels, A., Frame, M. C. \& Brunton, V. G. E-cadherin-integrin crosstalk in cancer invasion and metastasis. J. Cell Sci. 126, 393-401 (2013).

31. Thomas, L. W., Lam, C. \& Edwards, S. W. Mcl-1; the molecular regulation of protein function. FEBS Lett. 584, 2981-2989 (2010).

32. Lee, W. S. et al. Myeloid cell leukemia-1 promotes epithelial-mesenchymal transition of human gastric cancer cells. Oncol. Rep. 34, 1011-1016 (2015).

33. Wang, J. M. et al. The antiapoptotic gene mcl- 1 is up-regulated by the phosphatidylinositol 3-kinase/Akt signaling pathway through a transcription factor complex containing CREB. Mol. Cell Biol. 19, 6195-6206 (1999).

34. Booy, E. P., Henson, E. S. \& Gibson, S. B. Epidermal growth factor regulates Mcl1 expression through the MAPK-Elk-1 signalling pathway contributing to cell survival in breast cancer. Oncogene 30, 2367-2378 (2011).

35. Inuzuka, H. et al. SCF(FBW7) regulates cellular apoptosis by targeting MCL1 for ubiquitylation and destruction. Nature 471, 104-109 (2011).

36. Schmid, M. C. et al. Receptor tyrosine kinases and TLR/L1Rs unexpectedly activate myeloid cell PI3kgamma, a single convergent point promoting tumor inflammation and progression. Cancer Cell 19, 715-727 (2011).

37. Hernandez, C. et al. Progastrin represses the alternative activation of human macrophages and modulates their influence on colon cancer epithelial cells. PloS ONE 9, e98458 (2014).

38. Kong, L. Q. et al. The clinical significance of the CD163+ and CD68+ macrophages in patients with hepatocellular carcinoma. PloS ONE 8, e59771 (2013).

39. Chen, Y., Zhang, S., Wang, Q. \& Zhang, X. Tumor-recruited M2 macrophages promote gastric and breast cancer metastasis via M2 macrophage-secreted CHI3L1 protein. J. Hematol. Oncol. 10, 36 (2017).

40. Cui, Y. L., Li, H. K., Zhou, H. Y., Zhang, T. \& Li, Q. Correlations of tumor-associated macrophage subtypes with liver metastases of colorectal cancer. Asian Pac. J. cancer Prev. 14, 1003-1007 (2013).

41. Nagorsen, D. et al. Tumor-infiltrating macrophages and dendritic cells in human colorectal cancer: relation to local regulatory $T$ cells, systemic T-cell response against tumor-associated antigens and survival. J. Transl. Med. 5, 62 (2007).

42. Mitra, A., Mishra, L. \& Li, S. Technologies for deriving primary tumor cells for use in personalized cancer therapy. Trends Biotechnol. 31, 347-354 (2013).

43. Zheng, W. \& Pollard, J. W. Inhibiting macrophage PI3Kgamma to enhance immunotherapy. Cell Res. 26, 1267-1268 (2016).

44. Le, D. T. et al. PD-1 blockade in tumors with mismatch-repair deficiency. $N$. Engl. J. Med. 372, 2509-2520 (2015).

45. Overman, M. J. et al. Nivolumab in patients with metastatic DNA mismatch repair-deficient or microsatellite instability-high colorectal cancer (CheckMate 142): an open-label, multicentre, phase 2 study. Lancet Oncol. 18, 1182-1191 (2017).

46. Overman, M. J. et al. Durable clinical benefit with nivolumab plus ipilimumab in DNA mismatch repair-deficient/microsatellite instability-high metastatic colorectal cancer. J. Clin. Oncol. 36, 773-779 (2018). 